\title{
Limits of applicability of the advection-dispersion model in aquifers containing connected high-conductivity channels
}

\author{
Gaisheng Liu and Chunmiao Zheng \\ Department of Geological Sciences, University of Alabama, Tuscaloosa, Alabama, USA
}

Steven M. Gorelick

Department of Geological and Environmental Sciences, Stanford University, Stanford, California, USA

Received 5 October 2003; revised 14 May 2004; accepted 3 June 2004; published 26 August 2004.

[1] The macrodispersion model from stochastic transport theory is demonstrated to be of limited utility when applied to heterogeneous aquifer systems containing narrow connected pathways. This is so even when contrasts in hydraulic conductivity $(\mathrm{K})$ are small and variance in $\ln \mathrm{K}$ is less than 0.10 . We evaluated how well an advectiondispersion model (ADM) could be used to represent solute plumes transported through mildly heterogeneous three-dimensional (3-D) systems characterized by a well-connected dendritic network of $10 \mathrm{~cm}$ wide high-K channels. Each high-K channel network was generated using an invasion percolation algorithm and consisted of $\sim 10 \%$ by volume high-K regions. Contrasts in $\mathrm{K}$ between the channels and matrix were varied systematically from $2: 1$ to $30: 1$, corresponding to $\ln \mathrm{K}$ values ranging from 0.04 to 1.05 . Simulations involved numerical models with 3-D decimeter discretization, and each model contained 2-4 million active cells. Transport through each channel network considered only the processes of advection and molecular diffusion. In every case, the temporal change in the second spatial moment of concentrations was linear, with $\mathrm{R}^{2}$ values ranging from 0.97 to 0.99 . The third spatial moment, or alternatively, the skewness coefficient values, indicated significant tailing downstream of the plume center. For each case, a corresponding ADM was used to simulate transport through the system. The corresponding ADM employed the effective mean hydraulic conductivity that reproduced the total discharge through the channel network system under an identical ambient gradient. Dispersivity values used in the ADM were obtained from the temporal change in the second spatial moments of concentrations for the plumes in the channel network systems and ranged from $0.014 \mathrm{~m}$ to $0.85 \mathrm{~m}$. The results indicate that as the conductivity contrast between the channels and matrix increased, the simulated plumes in the channel network system became more and more asymmetric, with little solute dispersed upstream of the plume center and extensive downstream spreading of low concentrations. Distinctly different spreading was found upstream versus downstream of the plume center. The ADM failed to capture this asymmetry. Comparison of each plume in the channel network system with the corresponding plume produced using the corresponding ADM showed a maximum correlation of only 0.64 and a minimum fractional error of 0.29 for cases in which the $\log \mathrm{K}$ variance was $\sim 0.20$ (ln $\mathrm{K}$ variance was $\sim 1.0$ ). At early times the correlations were as low as 0.40 . The greatest correlation occurred at late times and for cases in which a wide source was considered. INDEX TERMS: 1832 Hydrology: Groundwater transport; 1831 Hydrology: Groundwater quality; 1829 Hydrology: Groundwater hydrology; KEYWORDS: aquifer heterogeneity, solute transport, stochastic hydrogeology, advection-dispersion, percolation theory, Macrodispersion Experiment (MADE) site

Citation: Liu, G., C. Zheng, and S. M. Gorelick (2004), Limits of applicability of the advection-dispersion model in aquifers containing connected high-conductivity channels, Water Resour. Res., 40, W08308, doi:10.1029/2003WR002735.

\section{Introduction}

[2] The applicability of the classical advection dispersion model (referred to here as the ADM) to describe solute behavior in highly heterogeneous aquifers has remained an

Copyright 2004 by the American Geophysical Union. 0043-1397/04/2003WR002735\$09.00 open issue since stochastic transport theory was first presented [Gelhar et al., 1979; Dagan, 1984; Neuman and Zhang, 1990]. Although it is evident that solute migration observed in the field often cannot be represented using the macrodispersion approach [Carrera, 1993], many of the underlying reasons for this "anomalous" behavior have been neither fully conceptualized nor quantified. Macrodispersion theory is based on the premise that groundwater 
velocity variations are due to heterogeneity in hydraulic conductivity, $\mathrm{K}$, which is assumed to be correlated but otherwise random. From a geologic perspective, this premise is often unjustified because connectedness rather than randomness is expected in heterogeneous aquifers [Koltermann and Gorelick, 1996].

[3] Fogg et al. [2000] and LaBolle and Fogg [2001] were among the first to simulate "anomalous" transport behavior from a geologic perspective. Their 3-D hydrofacies model of the aquifer underlying Lawrence Livermore National Laboratory (LLNL) represented connected highconductivity (referred to as high-K) channel deposits at the meter scale. Using flow simulation, Fogg et al. [2000] demonstrated the influence of connected heterogeneity on pump test response. Using the same hydrofacies model to represent aquifer heterogeneity at the LLNL site, LaBolle and Fogg [2001] showed that solute migration depended on connected hydrostratigraphic features and molecular diffusional exchange. In the recent work by Zinn and Harvey [2003], flow and transport behaviors were compared in 2-D conductivity fields in which there were high-, intermediate-, or low-conductivity connected regions. They found substantially different flow and transport behaviors in the three different conductivity fields. This was so despite the fact that each conductivity field had nearly identical lognormal univariate conductivity distributions, and nearly identical spatial covariances. Connected preferential flow channels were also found to influence groundwater flow and solute dispersion over a short distance in dipole forced gradient tracer tests [Tiedeman and Hsieh, 2004]. Tiedeman and Hsieh [2004] showed that dispersivity estimates can differ depending on whether or not a continuous high-velocity path is formed between the two closely spaced wells. For a large well separation distance a continuous high-velocity path may not exist and the intrawell low-velocity region acts to reduce the variability of dispersivity values.

[4] Motivated by prior analysis of the MADE site data, where diffusive mass transport was shown to be a governing transport process [Feehley et al., 2000; Harvey and Gorelick, 2000; Julian et al., 2001], we adopt the notion of fine-scale geologic controls and build on the work of Zheng and Gorelick [2003], which explored in 2-D the significance of decimeter wide high-K channels on solute transport. We explore the conditions under which classical advection-dispersion theory is, or is not, applicable when decimeter wide connected high-K flow paths are present. Our assertion is that well-connected high-K networks can dominate subsurface transport in a manner that cannot be described by an ADM. Previous analyses have shown that decimeter wide connected pathways can determine the characteristics of solute migration, including plume shape and spreading [Feehley et al., 2000; Harvey and Gorelick, 2000; Zheng and Gorelick, 2003]. Despite comprising only a small percentage of an aquifer's volume, connected high-K networks produce asymmetric, non-Gaussian plumes with near-source peaks and extensive spreading in the direction of flow.

[5] We consider 3-D heterogeneity whose structure is not simply random and correlated, but rather consists of connected networks of aquifer material that act as preferential groundwater flow paths. Here, each high-K path, or channel, is on the order of a decimeter wide, and the dendritic network cannot be explicitly represented in a field-scale model typically with a grid spacing of meters or larger. This is because the channels are too narrow, and the channel locations cannot be identified as the geometry of the channel network is too complicated. Decimeter-scale channels are believed to occur at the MADE site and are believed to be responsible for the "anomalous" transport behavior over several hundreds of meters observed during two natural gradient tracer experiments.

[6] The purpose of this study is to investigate the basic characteristics of solute transport in flow fields controlled by connected high-K channels at the decimeter scale. The main topic we address is under what conditions, if any, does a 3-D advection-dispersion model based on macrodispersion theory adequately simulate transport in aquifers where narrow, connected, high-K pathways are present but not explicitly represented.

[7] The remainder of this paper is organized as follows. We begin by describing the generation of 3-D high-K channel networks using invasion percolation theory. Then, we discuss the numerical flow and transport models used to simulate transport through the synthetic channel network systems. Next, we discuss the methodologies used to evaluate transport behavior in the channel networks, the approach to development of the corresponding ADM, and the measures used in our comparisons. Finally, we present the results of large-scale 3-D numerical experiments that provide insight into transport behavior and help answer the fundamental question posed above regarding the range of applicability of the ADM.

\section{Channel Network Generation}

[8] We developed an invasion percolation algorithm that extends the one presented by Stark [1991] to generate 3-D connected high-K networks. Three steps are involved: (1) create an initial random K field, (2) based on Stark's invasion percolation algorithm, generate channels located preferentially in the high-K cells, and (3) assign binary $\mathrm{K}$ values, a high- $\mathrm{K}$ value assigned to channels and a low- $\mathrm{K}$ value assigned to the remaining nonchannel region.

[9] The generated high-K network patterns are fractaland scale-invariant. The arrangements produced are easiest to visualize as 3-D dendritic channel networks. The invasion percolation algorithm employs a regular grid. Each cell is assigned a random number that represents the likelihood of that cell to become a channel. A number of seed sites are assigned as starting points. The seeds can be placed anywhere and represent channels of unspecified stream order. If the seeds are placed at the downstream end of the system, a single seed cell would represent the outlet of a single stream, and a line of seeds would represent a preexisting stream into which others flowed. A channel network is formed by beginning at the seed locations, and tracing the sequence of weakest cell resistances (i.e., largest cell conductivities), as all locations emanating from the seed locations are explored. The algorithm is a mathematical process of channel growth that continues until the network of channels is established and no more channels form.

[10] Few geometric rules are imposed on the network during the growth process, but one is significant. A nonlooping condition is imposed during channel growth. This 
condition prevents geometries in which a particular channel discharges into itself. However, the condition also means that channels can have tributaries but cannot have distributaries. The nonlooping condition is implemented by simply requiring that a cell only can become part of the channel if any of its neighboring cells is not occupied by a channel.

[11] The distribution of channels depends on the initial assignment of cell conductivity. In practice, the conductivity field is random, and either normally or lognormally distributed. The drainage patterns generated tend to mimic those found in nature when structural controls are absent. From the viewpoint of a geologist, the resulting drainage patterns are realistic and likely are more representative of many natural patterns of connectivity than those produced statistically as second-order stationary random fields. The percolation network can be conditioned to the local presence or absence of a channel, but the procedure is essentially a heuristic algorithm that is not physically mechanistic. As such, the approach is inherently limited, but no more so than most geostatistical methods. Ideally, the initial conductivity field should be generated in a manner that respects those factors controlling the development of drainage patterns, such as lithology, structure, hydrologic and climatic characteristics, topography, and land cover [Rodriguez and Rinaldo, 1997]. That challenge remains.

[12] The invasion percolation algorithm developed for this study introduces two new features. First, our approach generates 3-D networks, and is not limited to 2-D as in the work of Stark [1991]. This enables us to consider networks of connected pathways that develop both vertically and horizontally. The degree of vertical connectivity can be controlled by introducing anisotropic correlation in the initially generated conductivity field. Stratigraphically, some vertical connectivity is expected, as erosion and deposition do not lead to perfectly horizontal layers. Second, highly dense networks result from the percolation approach when many seed locations are chosen. It is not uncommon for regions to be saturated with generated channels. Therefore we introduce a prespecified cutoff value that discourages the formation of excessive channels. Without the cutoff, channels are emplaced where the highest conductivity cells are identified during a particular step in the generation process. With the cutoff, channel development occurs only when the cell conductivities are larger than the cutoff value. The cutoff value can be specified based on the mean and variance of the initial cell conductivity field, or any other criteria. In addition, a postnetwork generation pruning step is performed to remove low-order channels as in the work of Stark [1991]. The Shreve stream ordering system is adopted in this work, and the lowest four channel orders were considered trivial and removed.

[13] The initial conductivity field can be generated or derived stochastically based on values measured at a field site. Here the initial K-field is assumed lognormally distributed and generated using the direct Fourier transform method [Robin et al., 1993]. The correlation length for the isotropic exponential variogram used in this work is $10 \mathrm{~cm}$, which maximizes the randomness in the initial K-field. The $\log -\mathrm{K}$ field generated is defined by its arithmetic mean, variance and correlation length. Using the percolation network algorithm, the connected high-K pathways are identified and then converted into channels. The remaining nonchannel region is treated as low- $\mathrm{K}$ matrix.

\section{Model Development}

\subsection{Governing Equations}

[14] The steady state 3-D movement of groundwater of constant density through porous media can be written as,

$$
\frac{\partial}{\partial x}\left(K_{x x} \frac{\partial h}{\partial x}\right)+\frac{\partial}{\partial y}\left(K_{y y} \frac{\partial h}{\partial y}\right)+\frac{\partial}{\partial z}\left(K_{z z} \frac{\partial h}{\partial z}\right)+q_{s}=0
$$

where $h$ is hydraulic head; $x, y$ and $z$ are the spatial coordinates; $K_{x x}, K_{y y}$, and $K_{z z}$ are the principal components of the hydraulic conductivity tensor in the $x, y$, and $z$ directions; and $q_{s}$ is the fluid sink/source.

[15] The transport of a conservative solute in 3-D groundwater flow is given by the advection-dispersion equation, which is the foundation of the ADM,

$$
\frac{\partial(n C)}{\partial t}=\frac{\partial}{\partial x_{i}}\left(n D_{i j} \frac{\partial C}{\partial x_{j}}\right)-\frac{\partial}{\partial x_{i}}\left(q_{i} C\right)+q_{s} C_{s} \quad i, j=1,2,3
$$

where $C$ is the solute concentration; $n$ is the effective porosity; $D_{i j}$ is the hydrodynamic dispersion tensor; $q_{i}$ is the $i$ component of the specific discharge or Darcy flux in the 1 , 2, 3 coordinate directions; and $C_{s}$ is the concentration in a fluid source/sink. The hydrodynamic dispersion tensor, $D_{i j}$, in an isotropic medium, with an accommodation made for different orthogonal transverse dispersivity values, can be expressed as [Burnett and Frind, 1987],

$$
\begin{gathered}
D_{x x}=\left(\alpha_{L} v_{x}^{2}+\alpha_{T H} v_{y}^{2}+\alpha_{T V} v_{z}^{2}\right) /|v|+D^{*} \\
D_{y y}=\left(\alpha_{L} v_{y}^{2}+\alpha_{T H} v_{x}^{2}+\alpha_{T V} v_{z}^{2}\right) /|v|+D^{*} \\
D_{z z}=\left(\alpha_{L} v_{z}^{2}+\alpha_{T V} v_{x}^{2}+\alpha_{T V} v_{y}^{2}\right) /|v|+D^{*} \\
D_{x y}=D_{y x}=\left(\alpha_{L}-\alpha_{T H}\right) v_{x} v_{y} /|v| \\
D_{x z}=D_{z x}=\left(\alpha_{L}-\alpha_{T V}\right) v_{x} v_{z} /|v| \\
D_{y z}=D_{z y}=\left(\alpha_{L}-\alpha_{T V}\right) v_{y} v_{z} /|v|
\end{gathered}
$$

where $v_{x}, v_{y}$, and $v_{z}$ are the components of the seepage velocity and $|v|$ is its magnitude; $\alpha_{L}$ is the longitudinal dispersivity; $\alpha_{T H}$ and $\alpha_{T V}$ are the transverse dispersivities in the horizontal and vertical directions, respectively, and $D^{*}$ is the molecular diffusion coefficient in porous media.

\subsection{Numerical Model of Flow System}

[16] To explore flow and transport through heterogeneous media we solved equations (1) and (2) numerically for the system displayed in Figure 1. The large scale numerical model used a fine discretization and consisted of over 4 million active cells. The governing flow equation (equation (1)) was solved using Modflow-2000 [Harbaugh et al., 2000]. The flow domain is $200.0 \mathrm{~m}$ long by $51.2 \mathrm{~m}$ wide by $2.0 \mathrm{~m}$ thick. The discretization of each of the 20 layers is $10 \mathrm{~cm}$. Each layer consists of 512 columns and 430 rows. 


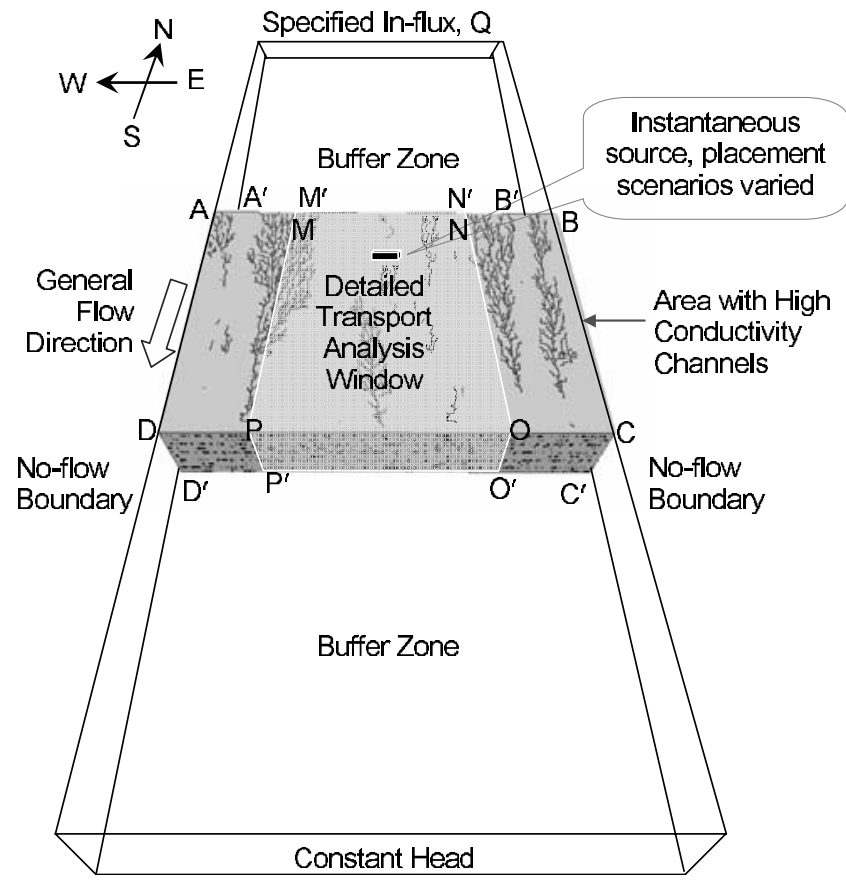

Figure 1. Schematic diagram of 3-D synthetic aquifer model.

The column spacing is constant at $10 \mathrm{~cm}$. The row spacing is $10 \mathrm{~cm}$ within the channel matrix region, but increases progressively toward the northern and southern boundaries. The generated high $\mathrm{K}$ channels are also $10 \mathrm{~cm}$ wide. The use of $10 \mathrm{~cm}$ wide cells to represent $10 \mathrm{~cm}$ wide channels is justified by two additional tests employing finer meshes (see section 5.2). A "window" in the central portion of Figure 1, consisting of over 2 million cells, is the submodel domain used for detailed analysis of transport. To minimize boundary effects, the window region is surrounded by two homogeneous buffer zones in the north and south, as shown in Figure 1. In addition, the detailed transport analysis window is located some distance away from the eastern and western boundaries.

[17] The synthetic aquifer has a constant saturated thickness. Flow originates at the vertical plane shown at the top of Figure 1 where the lateral flux $Q$ is specified. Flow exits through the vertical plane along the bottom of Figure 1, where a constant head boundary is established. There is no flow across the left and right vertical boundary planes, or through the base of the system. The specified flux $Q$ is determined such that an average hydraulic gradient of 0.003 is achieved. In the channel matrix region a uniform high $\mathrm{K}$ value is assigned to the channels and a lower uniform value is assigned to the matrix. The ratio between the channel and matrix $\mathrm{K}$ values is systematically increased and examined in different simulation scenarios. The uniform $\mathrm{K}$ value imposed in the homogeneous buffer zones is assigned the effective $\mathrm{K}$ of the channel matrix region, which is unique for each specific $\mathrm{K}$ contrast.

\subsection{Transport in the Detailed Analysis Region}

[18] Detailed solute transport analysis was conducted in the window region as shown in Figure 1. For the channel network system, the only transport processes considered are advection and molecular diffusion. Mechanical dispersion is not included because velocity variations due to heterogeneity are explicitly represented in the finely discretized model. All simulations employed a uniform effective porosity of 0.2 , and a molecular diffusion coefficient of $1.0 \times$ $10^{6} \mathrm{~cm}^{2} / \mathrm{s}$, which is a typical value for ions in freshwater. Zero concentration gradient boundary conditions were established along the 2D planar margins of the window region.

[19] Two different solute source configurations were investigated: (1) narrow source in which the initial mass was distributed in a small region of low $\mathrm{K}$ matrix and (2) partitioned source in which the initial mass was distributed nonuniformly in proportion to the respective $\mathrm{K}$ values in the source region that is about 17 times larger than the narrow source volume. The different source configurations correspond to different field situations that might be encountered. The "narrow source" represents a field situation where the solute mass is injected only into a low $\mathrm{K}$ region. The "partitioned source" represents the distribution of solutes in the aquifer where the channel receives a higher amount of injected mass than the matrix in proportion to the ratio of channel versus matrix $\mathrm{K}$ values. For instance, when the $\mathrm{K}$ contrast between the channel and matrix is $30: 1$, within the source region the initial mass in a channel was 30 times that in the low $\mathrm{K}$ matrix.

[20] To compare the patterns of plume development among different scenarios, we use normalized time, expressed as the fraction of one pore volume that would be displaced during purely advective transport in the window region. Normalized time, $t^{\prime}$, is calculated as

$$
t^{\prime}=\frac{t Q^{\prime}}{\forall}
$$

where $t$ is the actual simulation time; $\forall$ is the total pore volume of the window region; and $Q^{\prime}$ is the discharge through the window,

$$
Q^{\prime}=\frac{\overline{M N}}{\overline{A B}} Q
$$

$\overline{M N}, \overline{A B}$, and $Q$ are illustrated in Figure 1. In all cases the transport time horizon corresponds to the advective displacement of 0.25 pore volume. This is a time before any mass exits the window region. To convert relative time as expressed in equation (4) to an actual simulation time in a specific model, consider an example in which the ratio of $K_{\text {channel }} / K_{\text {matrix }}$ is $10: 1$. The actual values used in the model for $K_{\text {channel }}$ and $K_{\text {matrix }}$ are 2.4 and $0.24 \mathrm{~m} / \mathrm{d}$, respectively. The effective $\mathrm{K}$ of the channel matrix system is $0.37 \mathrm{~m} / \mathrm{d}$. The average seepage velocity is $0.0055 \mathrm{~m} / \mathrm{d}$. The window region for detailed transport analysis is $37 \mathrm{~m}$ long by $30 \mathrm{~m}$ wide by $2 \mathrm{~m}$ thick. The relative time of 0.25 pore volume corresponds to an actual simulation period of 1678.75 days.

[21] The transport model uses a highly accurate thirdorder TVD scheme for the advective transport terms, while the standard finite difference method is employed to solve all other terms. These features have been implemented in MT3DMS [Zheng and Wang, 1999], which was used here. To further insure numerical accuracy, the transport time step was bounded by a Courant number of 0.75 . The 2 million node transport submodel occupied only the window region, and a single simulation took tens of CPU hours on a 
Pentium 4-PC equipped with a $3.06 \mathrm{GHz}$ processor with $1 \mathrm{~Gb}$ of memory.

\section{Methods for Channel Network Transport Analysis}

[22] Our goal is to establish the conditions under which transport through media containing narrow connected pathways can be described using an ADM. To make this determination, for each of the two source configurations we simulated solute transport through heterogeneous aquifers consisting of high-K networks generated with the percolation network algorithm. We used various methods to evaluate the efficacy of the ADM to depict the plumes in the channel network systems. First, 3-D moment analysis was conducted to quantify plume migration and spreading and compare these spatial attributes and changes to Gaussian behavior. We introduce the third moments of a channel network plume as an important indicator of whether a channel network plume can be adequately simulated by an ADM. Second, we visually and statistically compare each 3 -D plume to that given by simulation using an ADM in which the dispersivities are based on stochastic transport theory using the change with time in the second spatial moment of concentration. To statistically evaluate the adequacy of an ADM to predict plume behavior in channel network systems, we consider two different measures, the mean fractional absolute residual (MFAR) and the linear correlation coefficient $(r)$, between a channel network plume and its corresponding ADM plume. MFAR describes the average error in concentration that occurs when the ADM is applied to predict the transport in the channel network system. The $r$, characterizes the degree of match or mismatch in the spatial structure between a plume in the channel network system and the corresponding ADM plume.

\subsection{Spatial Moment Analysis of Simulated Plumes}

[23] We use methods from stochastic transport theory to check whether plume transport behavior in the channel network system is consistent with advective-dispersive transport in which dispersivities are constant. This analysis of plume evolution relied, in part, on spatial moments of concentrations. The mnpth-order spatial moment of a concentration distribution, $M_{m n p}(t)$, is defined as [Freyberg, 1986]

$$
M_{m n p}(t)=\int_{-\infty}^{+\infty} \int_{-\infty}^{+\infty} \int_{-\infty}^{+\infty} n C(x, y, z, t) x^{m} y^{n} z^{p} d x d y d z
$$

The zeroth moment, $M_{000}(t)$, measures the total mass in the aquifer at time $t$. The first moments normalized by the total mass define the location of plume center $\left(x_{c}, y_{c}, z_{c}\right)$, or

$$
x_{c}=M_{100} / M_{000}, y_{c}=M_{010} / M_{000}, z_{c}=M_{001} / M_{000}
$$

The second moments, similarly normalized, measure the spatial covariance of a plume, $\sigma_{i j}^{2}$,

$$
\begin{array}{cc}
\sigma_{x x}^{2}=\frac{M_{200}}{M_{000}}-x_{c}^{2}, & \sigma_{y y}^{2}=\frac{M_{020}}{M_{000}}-y_{c}^{2}, \\
\sigma_{z z}^{2}=\frac{M_{002}}{M_{000}}-z_{c}^{2} & \sigma_{x y}^{2}=\sigma_{y x}^{2}=\frac{M_{110}}{M_{000}}-x_{c} y_{c}, \\
\sigma_{x z}^{2}=\sigma_{z x}^{2}=\frac{M_{101}}{M_{000}}-x_{c} z_{c}, & \sigma_{y z}^{2}=\sigma_{z y}^{2}=\frac{M_{011}}{M_{000}}-y_{c} z_{c}
\end{array}
$$

The third moments, through the skewness coefficient $\gamma$, describe the asymmetry of plume about its center [after Harvey and Gorelick, 1995],

$$
\begin{aligned}
& \gamma_{x}=\left[\frac{M_{300}}{M_{000}}-3 \frac{M_{100} M_{200}}{M_{000}^{2}}+2\left(\frac{M_{100}}{M_{000}}\right)^{3}\right] / \sigma_{x x}^{3} \\
& \gamma_{y}=\left[\frac{M_{030}}{M_{000}}-3 \frac{M_{010} M_{020}}{M_{000}^{2}}+2\left(\frac{M_{010}}{M_{000}}\right)^{3}\right] / \sigma_{y y}^{3} \\
& \gamma_{z}=\left[\frac{M_{003}}{M_{000}}-3 \frac{M_{001} M_{002}}{M_{000}^{2}}+2\left(\frac{M_{001}}{M_{000}}\right)^{3}\right] / \sigma_{z z}^{3}
\end{aligned}
$$

A value of zero indicates the plume is perfectly symmetric. Considering the plume geometry in the direction of flow $(y$ in our case), a positive value of skewness occurs when the plume peak is shifted upstream and the plume exhibits downstream tailing. As the magnitude of skewness grows larger, the plume becomes increasingly asymmetric.

[24] For each simulated plume we computed the spatial moments and their changes with time. In a steady state, uniform flow field with an instantaneous source, the hydrodynamic dispersion tensor, $D_{i j}$, in equation (3) can be related to the rate of change in the spatial variance of a solute plume [Freyberg, 1986],

$$
D_{i j}=\frac{1}{2} \frac{d \sigma_{i j}^{2}}{d t}
$$

Combining equations (10) and (3) yields the estimates of dispersivities for the corresponding ADM,

$$
\begin{gathered}
\alpha_{L}=\frac{1}{v_{y}}\left[\frac{1}{2} \frac{d \sigma_{y y}^{2}}{d t}-D^{*}\right], \alpha_{T H}=\frac{1}{v_{y}}\left[\frac{1}{2} \frac{d \sigma_{x x}^{2}}{d t}-D^{*}\right], \\
\alpha_{T V}=\frac{1}{v_{y}}\left[\frac{1}{2} \frac{d \sigma_{z z}^{2}}{d t}-D^{*}\right]
\end{gathered}
$$

\subsection{Approaches to Compare Channel Network and Advective-Dispersive Model Plumes}

[25] Each simulated plume in each channel network system was compared to the corresponding plume resulting from an ADM. The corresponding ADM in each case used an average velocity based on the effective hydraulic conductivity of that particular channel network system, and the dispersivities calculated from moment analysis equation (11). All other model features (e.g., source placement, boundary conditions, and ambient hydraulic gradient) were identical in the paired channel network system and corresponding ADM. Both statistical and visual comparisons were made between snapshots of plumes in the channel network systems and snapshots of plumes produced by the corresponding ADM.

[26] Two different statistical measures are used to compare the two types of 3-D plumes, those in the channel network system versus those produced by the corresponding ADM. MFAR quantifies the average discrepancy in concentration between the two types of 3-D plumes,

$$
\begin{aligned}
\operatorname{MFAR} & =\frac{1}{2} \sum_{k=1}^{N} \frac{\left|\left(C_{1, k}-C_{2, k}\right)\right| \times V_{k}}{M_{0}} \\
& =\frac{1}{2 \times N} \sum_{k=1}^{N} \frac{\left|\left(C_{1, k}-C_{2, k}\right)\right|}{C_{\text {ave }}}
\end{aligned}
$$


where $C_{1}$ and $C_{2}$ are the individual concentrations in the channel network system and those produced by the ADM at each location, $k$, respectively; $C_{a v e}$ is the average concentration, which is identical in the channel network system and in the ADM plume; $N$ is the total number of $C_{1}$ or $C_{2}$ values above a minimum threshold value. The threshold value was set relative to the initial concentration in the narrow source case and was fixed at $10^{-6}$. Below the threshold $10^{-6}$, the concentrations are considered indistinguishable from zero. $M_{0}$ is the total mass for each of the plumes in the pair under examination. $V_{k}$ is the pore water volume of each 3-D cell where concentrations are compared. The sum of absolute mass differences divided by the total mass, $M_{0}$, lies between 0 and 1 , where 0 represents a perfect match between the two plumes. The value 2 appears in MFAR to maintain an upper limit of $\operatorname{MFAR}=1$, and arises because $C_{a v e}$ is identical for the channel network and ADM plumes.

[27] Statistical comparisons between the spatial structure of each channel network plume and the corresponding plume produced by the ADM relied on the linear correlation coefficient of the log values of concentration. Our initial analyses showed the correlation coefficient to be dominated by the peak concentration values. Here our interest is the average correlation between all concentration pairs, and therefore log values were taken giving the correlation coefficient as:

$$
r=\frac{\sum_{k=1}^{N}\left(\log C_{1, k}-\overline{\log C_{1}}\right)\left(\log C_{2, k}-\overline{\log C_{2}}\right)}{\sqrt{\sum_{k=1}^{N}\left(\log C_{1, k}-\overline{\log C_{1}}\right)^{2}} \sqrt{\sum_{k=1}^{N}\left(\log C_{2, k}-\overline{\log C_{2}}\right)^{2}}}
$$

where $\overline{\log C_{1}}$ and $\overline{\log C_{2}}$ is the mean $\log$ concentration of the plume in the channel network systems and of the plume given by the ADM. Only those values above $10^{-6}$ of the initial source concentrations were considered in the computation of $r$. Because the ADM in this study yields Gaussian plumes, the $r$ value is essentially a measure of how closely the plumes in the channel network resemble a Gaussian spatial distribution. Although a perfect match, where $r=1.0$, would indicate no predictive loss when using the ADM, if some mismatch is acceptable, then a lower $r$ value can be adopted. For example, we suggest $r=0.7$ as a value above which the ADM provides an adequate approximation of transport through the channel network system.

[28] Visual comparisons were also made between the plumes given by the ADM and in the channel network system. These comparisons were based on the 2-D vertical average of the concentrations in each case,

$$
C_{2 D}(j, i)=\frac{\sum_{k=1}^{\mathrm{NLAY}} C(j, i, k) \times V(j, i, k)}{\sum_{k=1}^{\mathrm{NLAY}} V(j, i, k)}
$$

where $C_{2 D}(j, i)$ is the vertically averaged concentration of all cells $(j, i)$ in 2-D plane view; $V(j, i, k)$ and $C(j, i, k)$ are the pore volume and concentration for cell $(j, i, k)$, and NLAY is the total number of model layers, which is 20 in this study.

\section{Results}

[29] Using the methods described in section 4, we present the results from large-scale simulations involving decimeter
Table 1. Hydraulic Conductivity Field and Its Statistics at Different K Contrasts Examined in the Synthetic Aquifer Model ${ }^{\mathrm{a}}$

\begin{tabular}{lcccccc}
\hline \multirow{7}{*}{ K Ratio } & \multicolumn{2}{c}{ Model K Values } \\
\cline { 2 - 5 } & Channel & Matrix & Mean K & Var K & Mean (ln K) & Var (ln K) \\
\hline \multicolumn{7}{c}{ Channel Realization 1 } \\
$2: 1$ & 0.48 & 0.24 & 0.26 & 0.0046 & -1.36 & 0.040 \\
$3: 1$ & 0.72 & 0.24 & 0.28 & 0.019 & -1.33 & 0.10 \\
$5: 1$ & 1.20 & 0.24 & 0.33 & 0.077 & -1.28 & 0.22 \\
$10: 1$ & 2.40 & 0.24 & 0.44 & 0.39 & -1.22 & 0.44 \\
$20: 1$ & 4.80 & 0.24 & 0.66 & 1.73 & -1.15 & 0.75 \\
$30: 1$ & 7.20 & 0.24 & 0.88 & 4.03 & -1.12 & 0.96 \\
& & & & & & \\
$2: 1$ & 0.48 & 0.24 & 0.27 & 0.0052 & -1.36 & 0.043 \\
$3: 1$ & 0.72 & 0.24 & 0.29 & 0.021 & -1.32 & 0.11 \\
$5: 1$ & 1.20 & 0.24 & 0.34 & 0.083 & -1.27 & 0.23 \\
$10: 1$ & 2.40 & 0.24 & 0.46 & 0.42 & -1.20 & 0.48 \\
$20: 1$ & 4.80 & 0.24 & 0.70 & 1.88 & -1.13 & 0.81 \\
$30: 1$ & 7.20 & 0.24 & 0.94 & 4.38 & -1.08 & 1.05 \\
\hline
\end{tabular}

${ }^{\mathrm{a}} \mathrm{K}$ values are in $\mathrm{m} / \mathrm{d}$.

wide connected networks, for a range of contrasts in hydraulic conductivity, and with different selected source configurations. Scenarios involved a 3-D connected network with $\sim 9 \%$ high-K channels (realization 1 ), six sets of ratios of hydraulic conductivity values assigned to the channels versus to the matrix, $2: 1,3: 1,5: 1,10: 1,20: 1$ to $30: 1$, and two source placements, a narrow source and a partitioned source. The initial mass in each of the different simulations was equal to foster comparison. To evaluate the impact of spatial variability in the channel network system, a second high-K connected network realization (realization 2) was generated, and parallel transport simulations and plume analyses were conducted.

[30] The characteristics of the hydraulic conductivity field under different $\mathrm{K}$ contrasts and key statistics from spatial moment analysis and network-ADM plume comparison are summarized in Tables 1-3 for the two channel network realizations. In our investigations despite contrasts in $\mathrm{K}$ ranging from 2:1 to 30:1, the variance in ln $\mathrm{K}$ ranged from only 0.04 to 1.05 . Note that because the finite difference model uses average $\mathrm{K}$ values between cells, the heterogeneities actually involved in the numerical experiments are slightly less than that indicated by either the assigned $\mathrm{K}$ contrasts or the calculated variances.

\subsection{Narrow Source Configuration}

[31] Before we discuss the quantitative measures of plume evolution resulting from transport through the channel network system, we present a visual analysis and some simple observations. The channel network and location of the narrow source are illustrated in Figures $2 \mathrm{a}$ and $2 \mathrm{~b}$. Figures $2 \mathrm{c}$ and $2 \mathrm{~d}$ show the concentrations at $t^{\prime}=0.25$ for the $K_{\text {channel }} / K_{\text {matrix }}$ ratios of 10:1 and 30:1, respectively. The plumes in the channel network system exhibit an asymmetric, non-Gaussian form, and significant mass trapped near the source with extensive downstream spreading. The plume asymmetry increases with $K_{\text {channel }} / K_{\text {matrix }}$ contrast, which is readily explained. Given the narrow source placement, all the initial mass is distributed in the lowconductivity matrix. A large $K_{\text {matrix }} / K_{\text {channel }}$ contrast means that this mass is contained in a region of low velocity, and escape is controlled by matrix-limited slow advection, 
Table 2. Summary of the Rate of Change in the Second Moments With Time, $d \sigma_{y y}^{2} / d t$, the $\mathrm{R}^{2}$ Values for the Best Fit Line, and the Dispersivities Calculated From the Second Moments at Different Sources and K Contrasts ${ }^{\mathrm{a}}$

\begin{tabular}{|c|c|c|c|c|c|c|c|c|c|c|c|c|c|c|c|c|c|c|c|}
\hline \multirow[b]{3}{*}{ K Ratio } & \multirow{3}{*}{$\begin{array}{c}\text { Mean } \\
\text { Velocity, } \\
\text { m/d }\end{array}$} & \multicolumn{6}{|c|}{ Narrow Source } & \multicolumn{6}{|c|}{ Partitioned Source } & \multicolumn{6}{|c|}{ Calculated $\alpha_{L}, \mathrm{~m}$} \\
\hline & & \multicolumn{2}{|c|}{ Full } & \multicolumn{2}{|c|}{ Upper } & \multicolumn{2}{|c|}{ Lower } & \multicolumn{2}{|c|}{ Full } & \multicolumn{2}{|c|}{ Upper } & \multicolumn{2}{|c|}{ Lower } & \multicolumn{3}{|c|}{ Narrow Source } & \multicolumn{3}{|c|}{ Partitioned Source } \\
\hline & & $\frac{d \sigma_{y y}^{2}}{d t}$ & $\mathrm{R}^{2}$ & $\frac{d \sigma_{y y}^{2}}{d t}$ & $\mathrm{R}^{2}$ & $\frac{d \sigma_{y y}^{2}}{d t}$ & $\mathrm{R}^{2}$ & $\frac{d \sigma_{y y}^{2}}{d t}$ & $\mathrm{R}^{2}$ & $\frac{d \sigma_{y y}^{2}}{d t}$ & $\mathrm{R}^{2}$ & $\frac{d \sigma_{y y}^{2}}{d t}$ & $\mathrm{R}^{2}$ & Full & Upper & Lower & Full & Upper & Lower \\
\hline \multicolumn{20}{|c|}{ Channel Realization 1} \\
\hline $2: 1$ & 0.0039 & 0.013 & 0.99 & 0.004 & 0.98 & 0.009 & 0.98 & 0.013 & 1.0 & 0.004 & 0.98 & 0.009 & 0.99 & 0.015 & 0.003 & 0.010 & 0.014 & 0.003 & 0.009 \\
\hline $3: 1$ & 0.0041 & 0.034 & 0.98 & 0.01 & 0.98 & 0.025 & 0.96 & 0.033 & 1.0 & 0.01 & 0.98 & 0.023 & 0.99 & 0.039 & 0.010 & 0.028 & 0.038 & 0.010 & 0.026 \\
\hline $10: 1$ & 0.0055 & 0.29 & 0.98 & 0.083 & 0.98 & 0.22 & 0.97 & 0.28 & 1.0 & 0.09 & 0.99 & 0.2 & 0.99 & 0.26 & 0.074 & 0.20 & 0.25 & 0.079 & 0.18 \\
\hline $20: 1$ & 0.0073 & 0.78 & 0.99 & 0.24 & 0.99 & 0.58 & 0.97 & 0.84 & 1.0 & 0.27 & 0.99 & 0.61 & 0.99 & 0.53 & 0.16 & 0.40 & 0.57 & 0.18 & 0.42 \\
\hline $30: 1$ & 0.0090 & 1.3 & 0.99 & 0.39 & 0.98 & 1.0 & 0.97 & 1.5 & 1.0 & 0.49 & 0.99 & 1.1 & 0.99 & 0.74 & 0.22 & 0.56 & 0.85 & 0.27 & 0.62 \\
\hline \multicolumn{20}{|c|}{ Channel Realization 2} \\
\hline $2: 1$ & 0.0039 & 0.012 & 1.00 & 0.004 & 0.98 & 0.008 & 0.99 & 0.014 & 1.00 & 0.005 & 0.98 & 0.009 & 0.99 & 0.014 & 0.003 & 0.008 & 0.016 & 0.004 & 0.010 \\
\hline $3: 1$ & 0.0042 & 0.033 & 1.00 & 0.01 & 0.98 & 0.023 & 0.99 & 0.037 & 1.00 & 0.012 & 0.98 & 0.025 & 1.00 & 0.037 & 0.010 & 0.025 & 0.042 & 0.013 & 0.028 \\
\hline $5: 1$ & 0.0047 & 0.091 & 1.00 & 0.029 & 0.99 & 0.065 & 0.99 & 0.098 & 1.00 & 0.034 & 0.99 & 0.065 & 1.00 & 0.095 & 0.029 & 0.067 & 0.10 & 0.034 & 0.068 \\
\hline $20: 1$ & 0.0078 & 0.93 & 1.00 & 0.29 & 0.98 & 0.69 & 0.98 & 0.87 & 1.00 & 0.3 & 0.99 & 0.59 & 1.00 & 0.60 & 0.19 & 0.44 & 0.55 & 0.19 & 0.37 \\
\hline $30: 1$ & 0.0097 & 1.7 & 1.00 & 0.54 & 0.98 & 1.2 & 0.97 & 1.6 & 1.00 & 0.54 & 0.99 & 1.1 & 0.99 & 0.88 & 0.28 & 0.64 & 0.81 & 0.28 & 0.55 \\
\hline
\end{tabular}

${ }^{\text {a }}$ The units of $d \sigma_{y y}^{2} / d t$ are $\times 10^{-2} \mathrm{~m}^{2} / \mathrm{d}$. Results are calculated for the upstream and downstream plume halves as well as for the full plume.

molecular diffusion, and the local channel geometry in the source area. Any mass that does reach a high-K channel is swept away. Qualitative comparison of our results to those observed during the first two tracer tests at the MADE site [Boggs and Adams, 1992; Boggs et al., 1993], where the source was emplaced in a low-K zone, suggests similar transport mechanisms and geometric controls of high-K channels.

[32] The results of spatial moment analysis for a $K_{\text {channel }}$ $K_{\text {matrix }}$ ratio of 10:1 are plotted in Figure 3 for successive transport times. The zeroth moment (Figure 3a) indicates no loss of mass. The first moment (Figure $3 b$ ) generally indicates a constant seepage velocity, although slightly slower migration occurs at early time because the initial mass is predominantly in the low- $\mathrm{K}$ matrix. The average seepage velocity of $0.0057 \mathrm{~m} / \mathrm{d}$ based on Figure $3 \mathrm{~b}$ is consistent with the $0.0055 \mathrm{~m} / \mathrm{d}$ computed using the effective $\mathrm{K}$ and ambient hydraulic gradient.
[33] The second moments (Figure 3c) show the changes in spatial variance about the plume center in $-x,-y$ and $-z$ directions. Evolution of the longitudinal spreading of the plume is nearly constant at late times. The results of analysis of the second moment are summarized in Table 2 for realizations 1 and 2. A strong linear relationship is observed in the change of plume spatial variance with time in all of the cases we examined. The $\mathrm{R}^{2}$ value for the variance versus time best fit line always exceeds 0.97 . The change in the second moment in $-y$ direction with time is more linear in realization 2 than realization 1 , although the $\mathrm{R}^{2}$ values of the best fit lines exceed 0.97 . This is likely due to a wider and thicker source used in the realization 2 simulations $(1.1 \mathrm{~m}$ wide $\times 0.5 \mathrm{~m}$ thick in realization 2 versus $0.4 \mathrm{~m}$ wide $\times 0.4 \mathrm{~m}$ thick in realization 1 ).

[34] On the basis of the zeroth, first, and second moments alone, one might assume that an ADM would properly represent plume migration and spreading, and that the

Table 3. Summary of the Linear Correlation Coefficients ( $r$ ), the Mean Fractional Absolute Residuals (MFAR) Between the Channel Network and the Corresponding ADM Plumes, and the Skewness Coefficients of the Channel Network Plumes at 0.25 Pore Volume

\begin{tabular}{|c|c|c|c|c|c|c|c|c|c|c|c|c|c|c|}
\hline \multirow[b]{3}{*}{ K Ratio } & \multicolumn{6}{|c|}{ Narrow Source } & \multicolumn{6}{|c|}{ Partitioned Source } & \multicolumn{2}{|c|}{ Skewness at $t^{\prime}=0.25$} \\
\hline & \multicolumn{2}{|c|}{ Full } & \multicolumn{2}{|c|}{ Upper } & \multicolumn{2}{|c|}{ Lower } & \multicolumn{2}{|c|}{ Full } & \multicolumn{2}{|c|}{ Upper } & \multicolumn{2}{|c|}{ Lower } & \multirow{2}{*}{$\begin{array}{l}\text { Narrow } \\
\text { Source }\end{array}$} & \multirow{2}{*}{$\begin{array}{c}\text { Partitioned } \\
\text { Source }\end{array}$} \\
\hline & $r$ & MFAR & $r$ & MFAR & $r$ & MFAR & $r$ & MFAR & $r$ & MFAR & $r$ & MFAR & & \\
\hline \multicolumn{15}{|c|}{ Channel Realization 1} \\
\hline $2: 1$ & 0.79 & 0.15 & 0.85 & 0.16 & 0.87 & 0.14 & 0.74 & 0.15 & 0.83 & 0.16 & 0.87 & 0.14 & 0.65 & 0.69 \\
\hline $3: 1$ & 0.77 & 0.20 & 0.83 & 0.21 & 0.87 & 0.18 & 0.73 & 0.18 & 0.80 & 0.20 & 0.89 & 0.16 & 0.84 & 0.88 \\
\hline $5: 1$ & 0.73 & 0.25 & 0.81 & 0.28 & 0.85 & 0.23 & 0.71 & 0.20 & 0.79 & 0.22 & 0.89 & 0.18 & 0.97 & 0.95 \\
\hline $10: 1$ & 0.67 & 0.32 & 0.77 & 0.37 & 0.82 & 0.26 & 0.70 & 0.23 & 0.77 & 0.26 & 0.89 & 0.20 & 1.03 & 0.94 \\
\hline $20: 1$ & 0.64 & 0.38 & 0.71 & 0.45 & 0.81 & 0.31 & 0.66 & 0.26 & 0.75 & 0.31 & 0.88 & 0.21 & 0.98 & 0.92 \\
\hline $30: 1$ & 0.62 & 0.41 & 0.69 & 0.49 & 0.79 & 0.34 & 0.63 & 0.28 & 0.72 & 0.34 & 0.87 & 0.23 & 0.99 & 0.90 \\
\hline \multicolumn{15}{|c|}{ Channel Realization 2} \\
\hline $2: 1$ & 0.78 & 0.15 & 0.86 & 0.15 & 0.87 & 0.16 & 0.75 & 0.16 & 0.84 & 0.16 & 0.88 & 0.15 & 0.63 & 0.60 \\
\hline $3: 1$ & 0.77 & 0.19 & 0.84 & 0.20 & 0.88 & 0.19 & 0.75 & 0.18 & 0.81 & 0.20 & 0.89 & 0.17 & 0.84 & 0.73 \\
\hline $5: 1$ & 0.75 & 0.23 & 0.82 & 0.25 & 0.88 & 0.22 & 0.74 & 0.20 & 0.80 & 0.22 & 0.89 & 0.18 & 0.93 & 0.76 \\
\hline $10: 1$ & 0.72 & 0.29 & 0.78 & 0.33 & 0.88 & 0.25 & 0.71 & 0.23 & 0.79 & 0.25 & 0.88 & 0.20 & 0.93 & 0.76 \\
\hline $20: 1$ & 0.66 & 0.35 & 0.73 & 0.43 & 0.85 & 0.27 & 0.66 & 0.26 & 0.77 & 0.30 & 0.87 & 0.23 & 0.96 & 0.82 \\
\hline $30: 1$ & 0.62 & 0.38 & 0.70 & 0.48 & 0.81 & 0.28 & 0.64 & 0.29 & 0.74 & 0.34 & 0.87 & 0.23 & 0.99 & 0.85 \\
\hline
\end{tabular}


(a) High-K Network

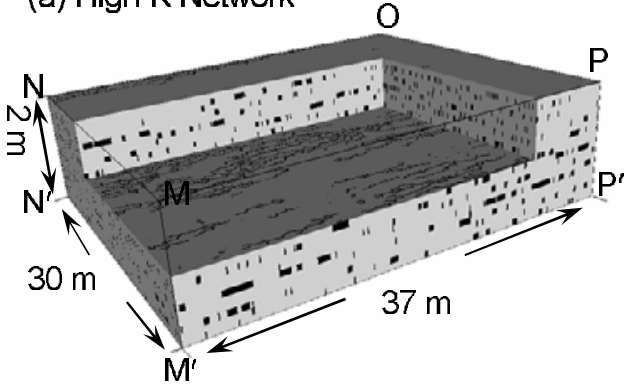

(c) K Ratio 10:1

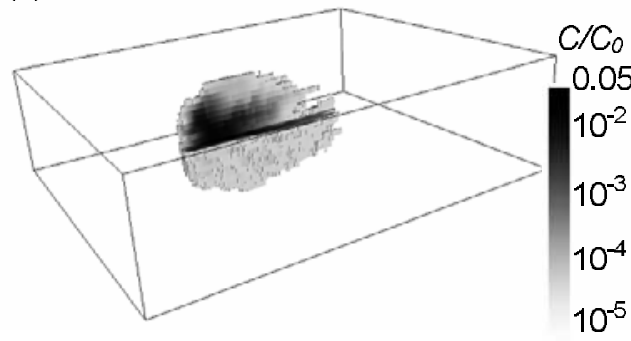

(b) Initial Narrow Source

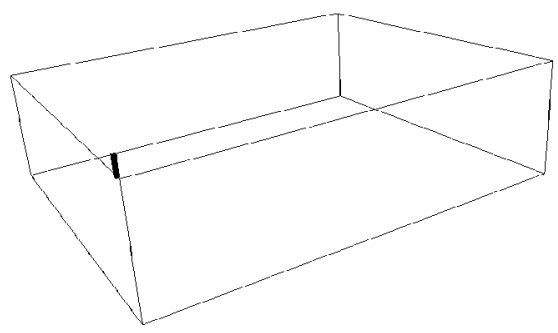

(d) K Ratio 30:1

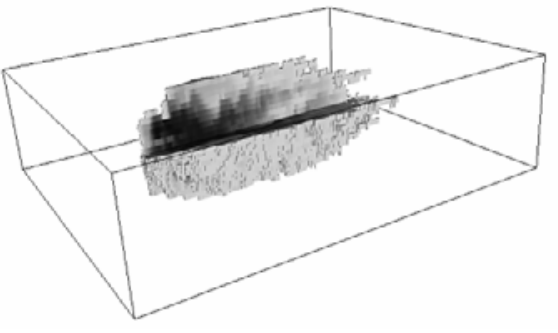

Figure 2. Calculated 3-D concentration distributions for narrow source case for different $\mathrm{K}$ contrast ratios $\left(K_{\text {channel }} / K_{\text {matrix }}\right)$ : (a) high-K network, (b) initial concentration, (c) $\mathrm{K}$ ratio $10: 1$ at 0.25 pore volume, and (d) $\mathrm{K}$ ratio $30: 1$ at 0.25 pore volume. Concentrations are normalized by the initial source concentration. Vertical exaggeration is $5: 1$.

applicable dispersivities based on the evolution of the second moments could be appropriately applied based on stochastic transport theory. The dispersivities based on the second moment appear in Table 2. Insight into the invalidity of this assumption can be obtained by inspecting the third moment. The components of the third moment in the $-x$, $-y$ and $-z$ directions (Figure $3 \mathrm{~d}$ ) or alternatively the skewness values (Table 3, realization 1) quantify the degree of asymmetry of each plume. At the initial time, the source is perfectly symmetric in all coordinate directions, as reflected by zero skewness at $t^{\prime}=0$. At later times, Figure $3 d$ shows significant plume tailing in the downstream direction corresponding to the large positive $y$ values of skewness. In contrast, the magnitude of the skewness coefficients in the $-x$ and $-z$ directions remains small over time. Similar trends in the spatial moments for simulations based on realization 2 are presented in Figures $3 \mathrm{e}-3 \mathrm{~h}$ for a $K_{\text {channel }} / K_{\text {matrix }}$ ratio of $10: 1$ and Table 3 (realization 2 ). In both realizations and for both source configurations the skewness value climbs with $\mathrm{K}$ contrast from $\sim 0.65$ to $\sim 0.95$. In all cases the plume peaks were displaced upstream of the average advective front. At $t^{\prime}=0.25$, the average plume peak displacements were approximately proportional to the $\mathrm{K}$ contrasts used in each scenario. The average peak displacements increased from $0.16 \mathrm{~m}$ to $0.97 \mathrm{~m}$ to $2.2 \mathrm{~m}$ for $\mathrm{K}$ contrasts of $2: 1,10: 1$, and $30: 1$ respectively, or $2 \%$ to $10 \%$ to $23 \%$ of the advective travel distance, in rough proportion to the $\mathrm{K}$ contrast. There was a general increase in this displacement versus the standard deviation of the plumes that ranged from 0.28 to 0.58 . That is, the plume peaks lagged the advective front by $\sim 1 / 4$ to $\sim 1 / 2$ of the spread of the plumes.

[35] The ADM for the conditions in our study yields symmetric Gaussian plumes. The third spatial moments are plotted in Figure 4 for different $K_{\text {channel }} / K_{\text {matrix }}$ contrasts at different relative times. For the ADM to be rigorously applicable, the third moments should all be zero. They are not. The magnitude of the third spatial moments provides a measure of how well a corresponding ADM can potentially match the transport affected by decimeter-scale preferential flow paths. Two features based on Figure 4 are prominent. First, the plumes become more asymmetric when the $K_{\text {channel }} / K_{\text {matrix }}$ contrast is increased, indicating that the $\mathrm{ADM}$ is a poorer model at higher $\mathrm{K}$ contrasts. Second, for a given $\mathrm{K}$ contrast, especially when the $\mathrm{K}$ contrast is large, plume symmetry deteriorates at early times but gradually improves with time. After a relative time of $t^{\prime}=0.15$, the plume symmetry stabilizes (Figure 4). Rigorously based on the third moments, an ADM is always inadequate when used to represent transport behavior in the channel network systems. The ADM never captured the skewness of plumes at any of the $\mathrm{K}$ ratios inspected, even when the variance of $\ln \mathrm{K}$ was as low as 0.040 and the $\mathrm{K}$ contrast was merely 2:1.

[36] We compared the 3-D plumes in the channel network system to those based on the ADM. Plume evolution was simulated with the ADM using equations (1)-(3), and dispersivities determined from equation (11) and the average seepage velocity based on the first moment. For example, in the simulation with $K_{\text {channel }} / K_{\text {matrix }}$ at $10: 1$, the dispersivity values calculated using equation (11) from stochastic transport theory are $\alpha_{L}=0.26 \mathrm{~m}, \alpha_{T H}=0.0039 \mathrm{~m}$, $\alpha_{T V}=0.0018 \mathrm{~m}$. These values are based on the average velocity, $v_{y}=0.0055 \mathrm{~m} / \mathrm{d}$, a molecular diffusion $8.64 \times$ $10^{-6} \mathrm{~m}^{2} / \mathrm{d}$, and the average changes in the spatial variance of concentration of $d \sigma_{y y}^{2} / d t=0.0029 \mathrm{~m}^{2} / \mathrm{d}, d \sigma_{x x}^{2} / d t=0.60 \times$ $10^{-4} \mathrm{~m}^{2} / \mathrm{d}$, and $d \sigma_{z z}^{2} / d t=0.37 \times 10^{-4} \mathrm{~m}^{2} / \mathrm{d}$.

[37] Statistical comparisons between the plumes produced by the ADM and those in the channel network system 
(a) Zeroth Moment, Realization 1

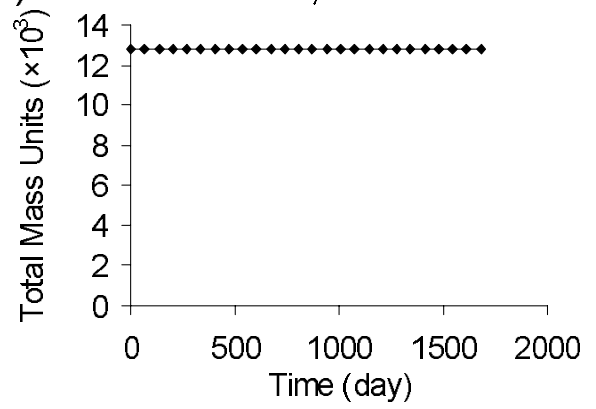

(c) Second Moments, Realization 1

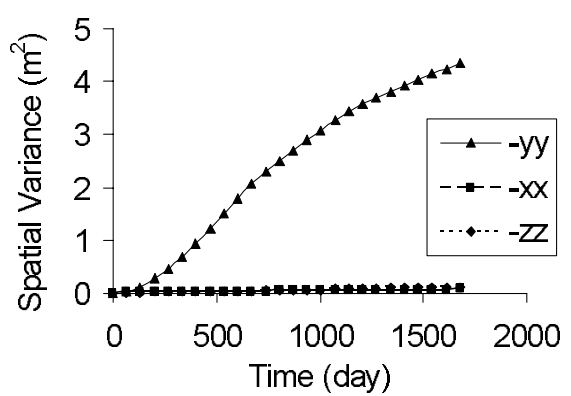

(e) Zeroth Moment, Realization 2

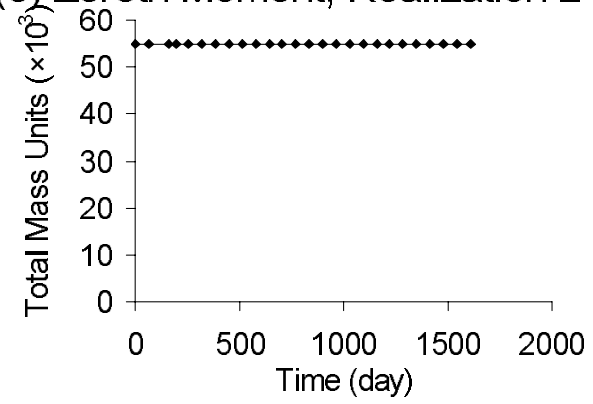

(g) Second Moments, Realization 2

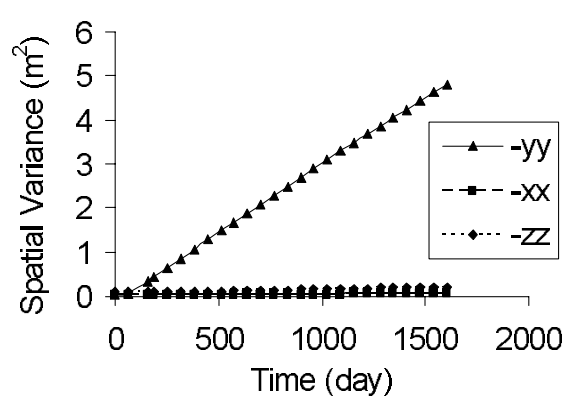

(b) First Moment, Realization 1

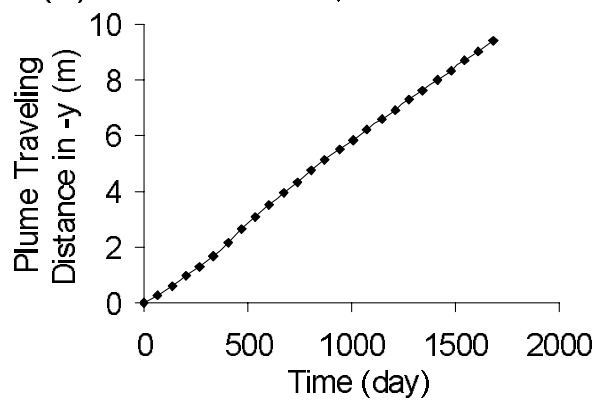

(d) Third Moments, Realization 1

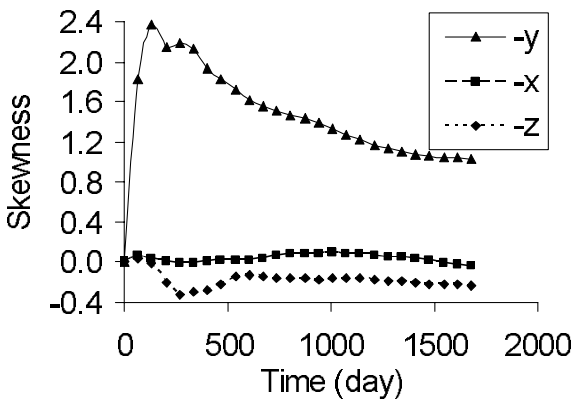

(f) First Moment, Realization 2

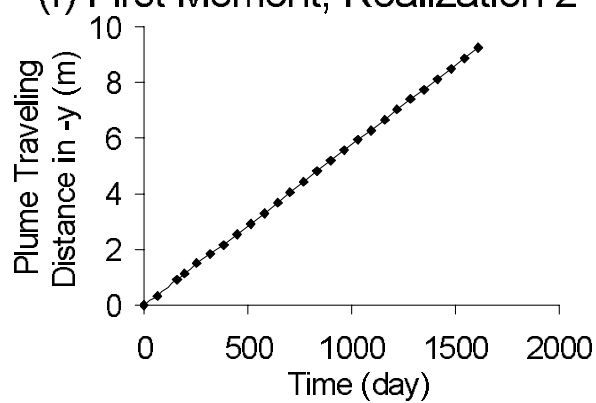

(h) Third Moments, Realization 2

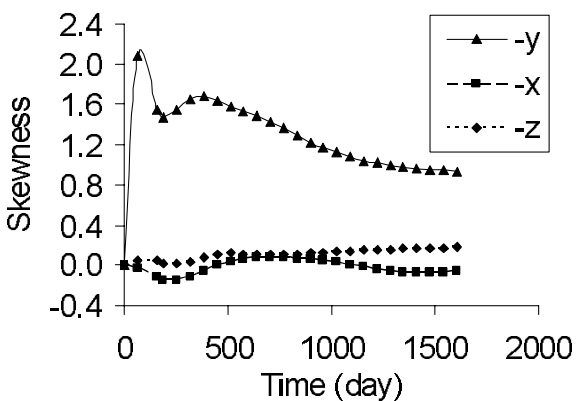

Figure 3. Spatial moment analysis results from the 3-D plumes in the channel network for a $\mathrm{K}$ contrast of 10:1 in the narrow source case: ( $a$ and e) zeroth moment, (b and f) first moment in ambient flow direction $y$, (c and $\mathrm{g}$ ) second moments in $-x x,-y y$, and $-z z$ directions, ( $\mathrm{d}$ and $\mathrm{h}$ ) third moments in $-x$, $-y$, and $-z$ directions. Figures $3 \mathrm{a}-3 \mathrm{~d}$ are for channel network realization 1 , and Figures $3 \mathrm{e}-3 \mathrm{~h}$ are for channel network realization 2 .

relied upon concentrations in 3-D, while visual comparisons relied on vertically integrated values given by equation (14). As mentioned earlier, only those concentrations above $\mathrm{C} / \mathrm{C}_{0}$ of $10^{-6}$ are considered in the following analysis. Both the 3-D concentrations in the channel network system, and the concentrations produced by the ADM were vertically averaged to produce Figure 5, which was used only for visual comparison. Figure 5 shows overlays of concentration contours from the ADM on the plumes in the channel network for each of the different $K_{\text {channel }} / K_{\text {matrix }}$ ratios.

[38] Despite some agreement, the ADM fails to represent two prominent features of transport behavior observed in the channel network system. First, the ADM underestimates the extensive solute spreading that results in low concen- 


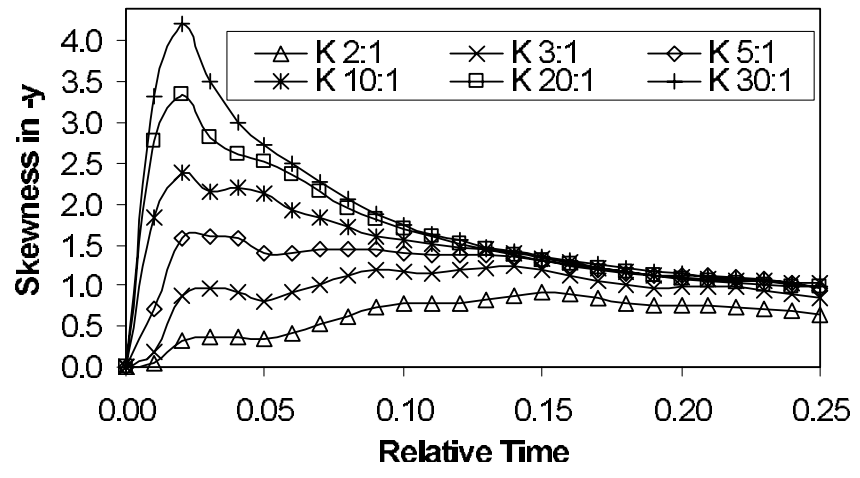

Figure 4. Plume skewness coefficients in the $-y$ direction for different $\mathrm{K}$ contrasts in the narrow source case.

trations downstream in each of the plumes in the channel network systems. Second, the ADM generates significant dispersion upstream of the plume as it migrates from the initial source plane. Because our simulations do not involve a permanent source, the excessive upstream spreading given by the ADM is not that commonly referred to as "back dispersion," but is a related phenomenon. With increasing $K_{\text {channel }} / K_{\text {matrix }}$ contrasts, these upstream and downstream discrepancies become more pronounced. Considering these disparities, the ADM fails to accurately represent solute transport behavior in any of the $K_{\text {channel }} / K_{\text {matrix }}$ contrasts investigated. However, the ADM may be an acceptable approximation when the $K_{\text {channel }} / K_{\text {matrix }}$ contrast is small and if the unrepresented features are unimportant in a particular application.

[39] Figure 6 shows the residuals between the channel network plumes and the corresponding plumes produced by the ADM. The residuals are calculated by subtracting the ADM concentration from that in the channel network systems in each model cell. The residual plots show that concentrations produced by the ADM upstream of the centroid plane of the migrating plume systematically exceed those observed in the channel network systems. A strong spatial relation can be observed between the residuals and the ADM plumes upstream. Downstream of the plume centroids, the ADM generally does a better job simulating the network plume, and the residuals are more randomly distributed. The downstream residuals are not obviously related to the network plumes or with the ADM concentrations.

[40] Statistical quantification of the average discrepancies between plumes in the channel network systems and the corresponding plumes produced using the ADM is displayed in Figure 7. The mean fractional absolute residual (MFAR) (Figure 7a) and linear correlation coefficient (Figure 7b) are shown for different relative advection times and different $K_{\text {channel }} / K_{\text {matrix }}$ contrasts. Values of these statistics are also reported in Table 3 for comparisons at $t^{\prime}=0.25$. The results of both statistical measures are consistent. Figure $7 \mathrm{a}$ shows that the overall error when using the ADM to represent transport behavior in the network system increases rapidly at early time but then gradually decreases. With increasing conductivity contrast, the error goes up dramatically. Figure $7 \mathrm{~b}$ indicates that the spatial correlation between the channel network and ADM plumes rapidly degrades with increasing conductivity contrast. At each K contrast, the correlation drops during early time and then asymptotes toward the end of the simulation time frame. Both measures show that the ADM, for which velocity variations due to $K$ heterogeneity are assumed random, is not representative of the "differential advection" between the low- $\mathrm{K}$ matrix and high- $\mathrm{K}$ channels. On the basis of a correlation of 0.7 the MFAR is about 0.3 , and the ADM fails as an acceptable approximation at $t^{\prime}=0.25$ when the $K_{\text {channel }} / K_{\text {matrix }} \geq 10: 1$.

[41] Simulations and comparisons to the ADM were carried out using channel network realization 2 with the narrow source configuration, and comparisons to the corresponding ADM were conducted. In addition to the different channel pattern in the two realizations, the (a) Source

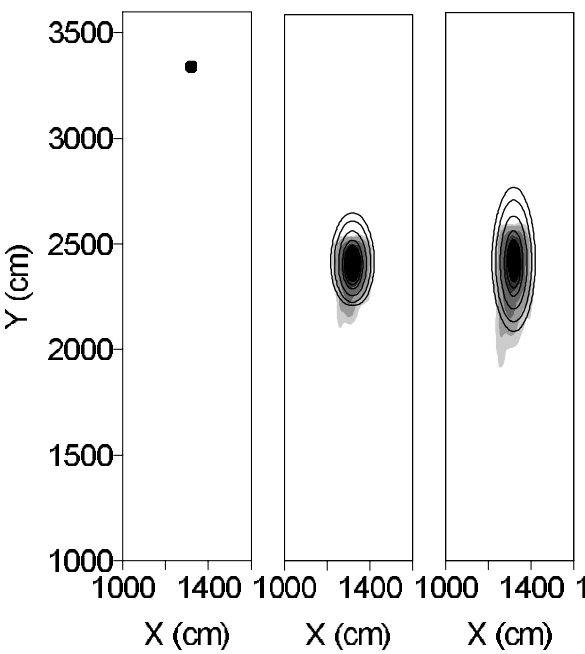

(d) $\mathrm{K} 5: 1$

(e) $\mathrm{K} 10: 1$ (f) $\mathrm{K} 20: 1$
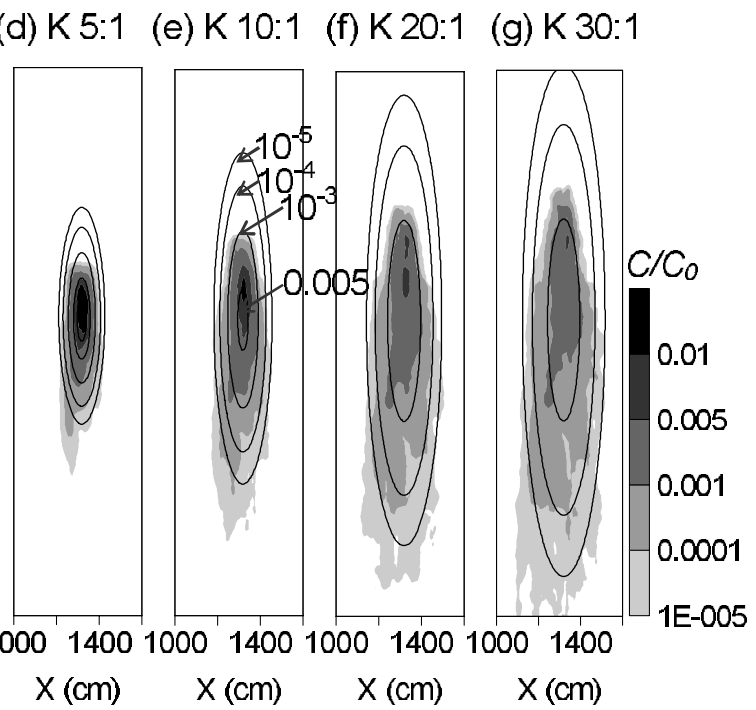

Figure 5. Comparison of the plumes in the channel network (shading) and from the corresponding ADM (contour lines) for different $\mathrm{K}$ contrasts in the narrow source case: (a) Source, (b) K 2:1, (c) K 3:1, (d) K 5:1, (e) K 10:1, (f) K 20:1, and (g) K 30:1. Plumes are shown for a simulation time of 0.25 pore volume. 

(a) K 2:1
(b) $\mathrm{K} 3: 1$
(c) K 5:1
(d) K 10:1
(e) K 20:1
(f) $\mathrm{K} 30: 1$

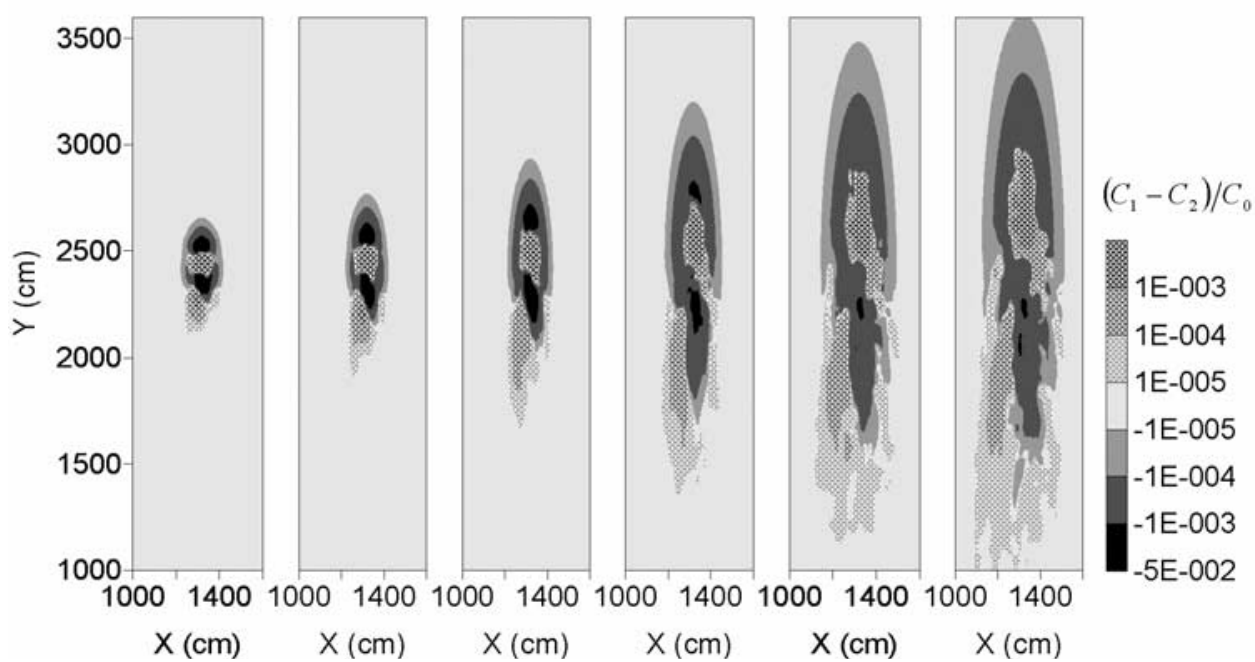

Figure 6. The network-ADM plume residuals for different $\mathrm{K}$ contrasts in the narrow source case: (a) K 2:1, (b) K 3:1, (c) K 5:1, (d) K 10:1, (e) K 20:1, and (f) $\mathrm{K} 30: 1$ at 0.25 pore volume.

channel fraction in realization 2 was $10 \%$, versus $9 \%$ in realization 2 . Results were very similar to those for the first channel network realization. The $r$ and MFAR values between the channel network and ADM plumes for the second realization are shown in Figures $7 \mathrm{c}$ and $7 \mathrm{~d}$. The curves for the two realizations, Figures $7 a-7 b$ and $7 c-7 d$, exhibit nearly identical characteristics despite the different high-K networks used in the synthetic aquifer. The major results from the second network realization are summarized in Tables 1-3.

\section{(a) MFAR, Narrow Source, Realization 1}

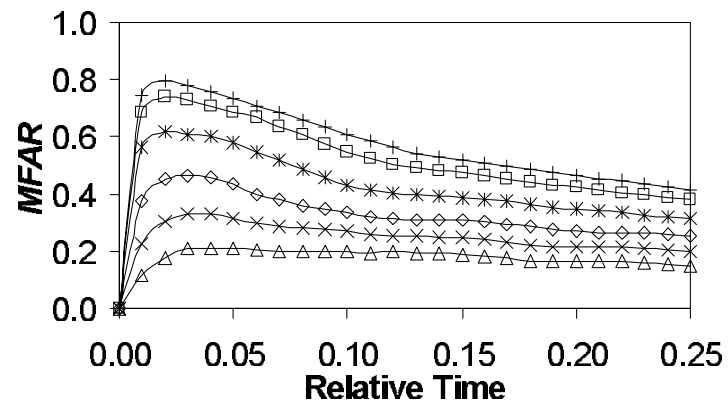

(c) MFAR, Narrow Source, Realization 2

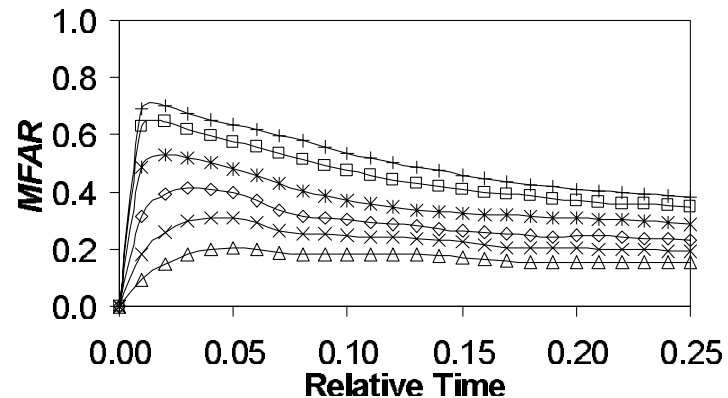

[42] Two distinct behaviors are observed between the upstream and downstream halves of plumes in the channel network systems (see Figure 5). Solute spreading upstream of the plume center is significantly less than that in the downstream area. To quantify the different transport behaviors between upstream and downstream, we conducted spatial moment analysis for the upstream and downstream plume halves separately. Dispersivities were also calculated for the two plume halves. The results are summarized in Table 2. As in the full plume case, the change of second

\section{(b) $r$, Narrow Source, Realization 1}
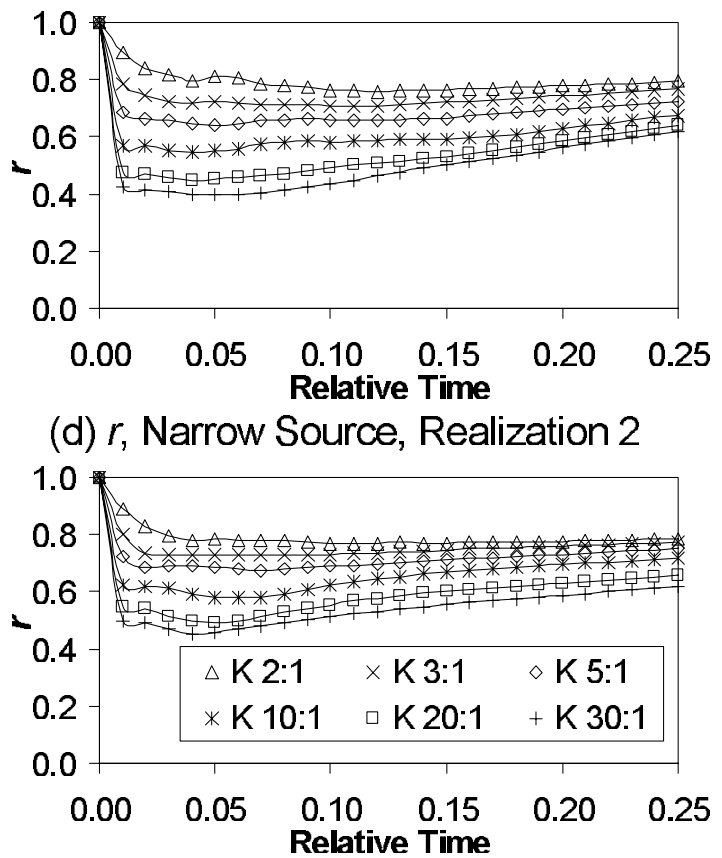

Figure 7. The mean fractional absolute residuals MFAR and linear correlation $r$ between the plumes in the 3-D channel network and from the corresponding ADM for different $\mathrm{K}$ contrasts in the narrow source case: (a) MFAR, realization 1, (b) $r$, realization 1, (c) MFAR, realization 2, and (d) $r$, realization 2 . The legend for different $\mathrm{K}$ contrasts is shown in Figure $7 \mathrm{~d}$. 
(a) MFAR, t' 0.25 , Narrow Source

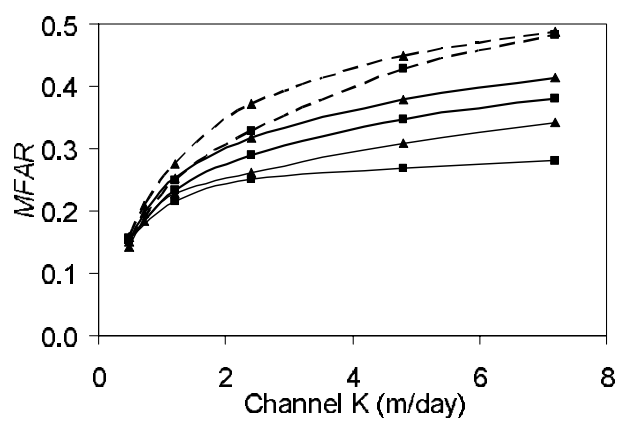

(c) MFAR, t' 0.25, Partitioned Source

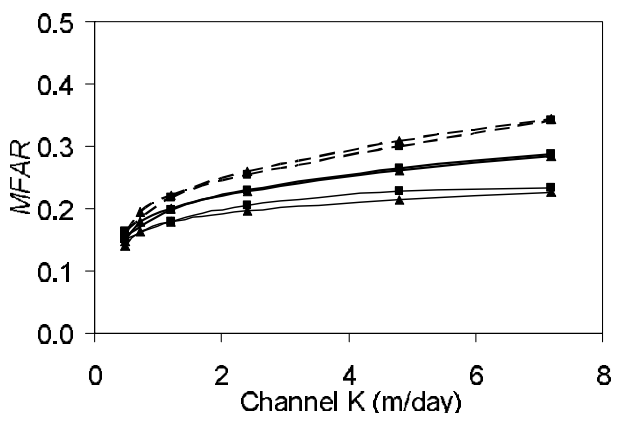

- Channel-Network Realization 1

- - Upstream Plume Half

_ Downstream Plume Half (b) $r, t^{\prime} 0.25$, Narrow Source

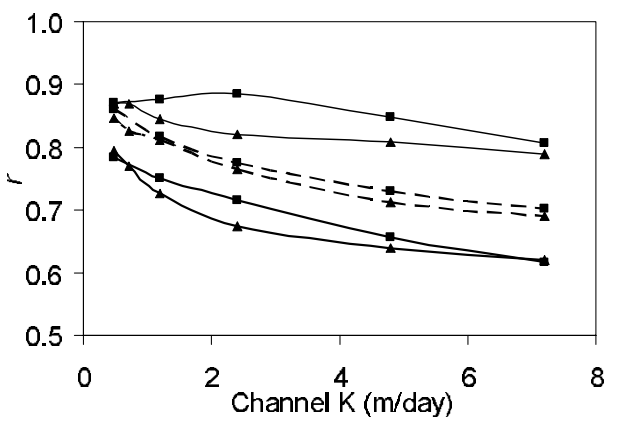

(d) $r$, t' 0.25, Partitioned Source

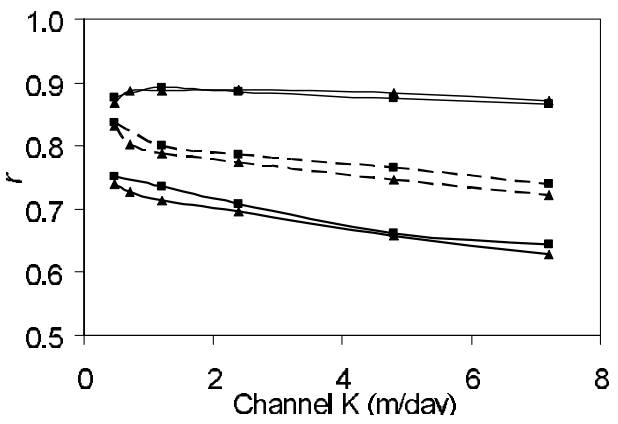

- Channel-Network Realization 2 Full Plume

Figure 8. MFAR and $r$ for the upstream plume half, downstream plume half, and full plume at different $\mathrm{K}$ contrasts and relative time 0.25: (a) MFAR, narrow source, (b) $r$, narrow source, (c) MFAR, partitioned source, and (d) $r$, partitioned source.

moments with time shows a strong linear trend for both upstream and downstream plume halves. However, the upstream and downstream linear trends differ as reflected in their contrasting dispersivity values. The downstream dispersivities are consistently $2.1 \sim 2.8$ times larger than the upstream dispersivities in all cases examined. It is interesting to note (Table 2) that the sum of each upstream and downstream dispersivity value is approximately equal to the dispersivity value computed for the full plume based on the temporal change in the second moments. This helps explain why a linear trend in the second moment is not in itself sufficient to determine a single dispersivity value for use in an ADM. Such a dispersivity value will not produce the asymmetric spreading behavior observed in the channel network system.

[43] Although the ADM failed to characterize either of the two halves of the plume individually, close inspection shows that the downstream portion of the plume is better represented than its upstream part. This can be seen in the residual plots (see Figure 6) where the upstream residuals have a spatial structure very similar to the ADM plumes and the downstream residuals are rather randomly distributed. To evaluate the efficacy of ADM in representing the upstream and downstream channel network transport behaviors separately, we computed the MFAR and $r$ for the two plume halves in addition to the full plumes. The results are summarized in Figures $8 \mathrm{a}$ and $8 \mathrm{~b}$ for different $K_{\text {channel }} / K_{\text {matrix }}$ contrasts and channel network realizations at $t^{\prime}=0.25$. The MFAR for the upstream plume halves is large for all $\mathrm{K}$ contrasts, indicating the ADM yields the largest error when simulating channel network transport in the upstream region. In contrast, the $\mathrm{ADM}$ provides a better representation for the transport in the channel network in the downstream region. The calculated $r$ values (see Figure $8 \mathrm{~b}$ ) show similar results. The spatial correlation between the channel network and ADM plumes upstream is much smaller than that downstream as the ADM provides a better representation of the spatial structure of the downstream portions of the plumes. The results of channel network realization 2 simulations are also included in Figures 8a and 8b. Despite some local differences, the results of the two realizations show consistent trends in the upstream versus downstream mismatch in the channel network versus ADM plumes.

[44] We mentioned earlier that ADM under the conditions examined in our study yields symmetric Gaussian plumes. Figure 9 compares the skewness of the channel network plumes and the corresponding plumes produced by the $\mathrm{ADM}$ for a $K_{\text {channel }} / K_{\text {matrix }}$ ratio of 10:1. Results indicate that the skewness coefficients of ADM plumes are close to zero at all different times. The plumes produced by the $\mathrm{ADM}$ are symmetric even at early times.

[45] To explore how diffusion and average seepage velocity affect transport behavior, two additional numerical experiments were conducted with the narrow source configuration: one in which the diffusion coefficient, $D^{*}$, was increased three fold, and another in which the hydraulic gradient, $i$, was decreased to one third of its original value. Both represent an increase in the relative importance of molecular diffusion compared to the base case. The 


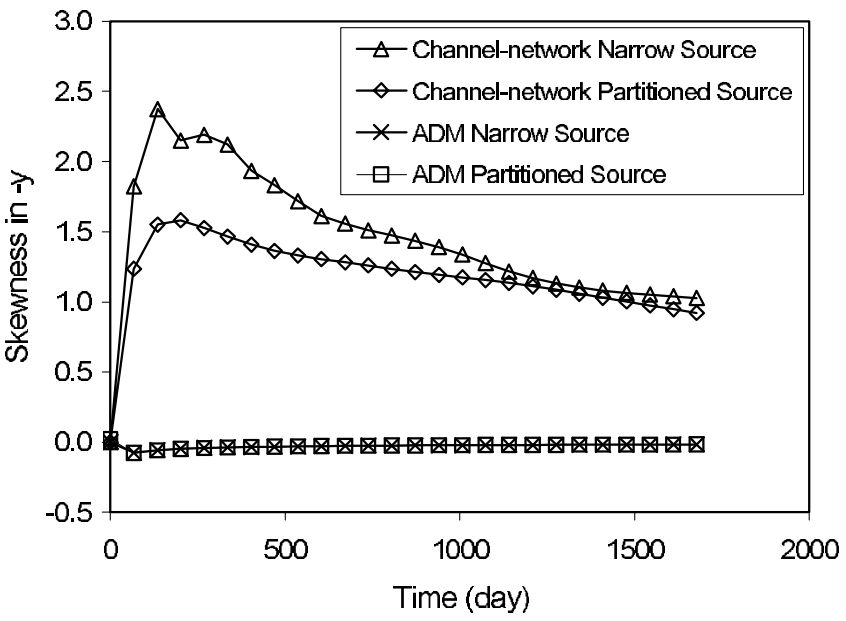

Figure 9. Comparison of the skewness coefficients for the plumes in the channel network with a K contrast of 10:1 and for the corresponding ADM plumes. Skewness coefficients are shown for both the narrow and partitioned source cases.

additional two experiments used a $K_{\text {channel }} / K_{\text {matrix }}$ ratio of 10:1, with all other physical conditions remaining identical to those in the original narrow source scenario. The potentially important role of molecular diffusive mixing has been noted by, for example, LaBolle and Fogg [2001] and Zinn and Harvey [2003]. In principle, mixing could cause transport through the channel network system to behave similarly to a corresponding system described by the ADM.

[46] The rate of temporal change in the (longitudinal) second moments of channel network plumes is $0.24 \times$ $10^{-2} \mathrm{~m}^{2} / \mathrm{d}$ for the two cases each involving an increase in the value of the molecular diffusion coefficient or a decrease in the hydraulic gradient, as compared to $0.29 \times 10^{-2} \mathrm{~m}^{2} / \mathrm{d}$ in the base case. This demonstrates that molecular diffusion actually reduces longitudinal spreading of plumes during transport through the channel network system. Figure 10 displays the correlation coefficient $r$ versus relative time for the two additional tests. Also shown is the base case in which neither value was altered. There is no appreciable difference in $r$ when either $D^{*}$ was increased or the hydraulic gradient was decreased. The correlation coefficient values for these two conditions could have been appreciably different, but they were not. However, when the two additional cases are compared to the base case, the correlation coefficients are significantly larger. This suggests that the resulting channel network plume can be better represented by the ADM if there is an increase in molecular diffusion or a decrease in the hydraulic gradient. It also shows that molecular diffusion plays a relatively important role in the system examined in this study.

\subsection{Partitioned Source Configuration}

[47] Here we discuss results from a second set of numerical experiments with a "partitioned source" in which the initial mass was allocated in proportion to the hydraulic conductivity values in the channel network system. Compared with the "narrow source" used in the first set of experiments, the partitioned source is about 17 times wider. Except for the source configuration, all other flow and transport conditions, including the high-K network inspected (i.e., realizations 1 and 2), were identical between this set and the first set of experiments.

[48] The location of the partitioned source is shown in Figure 11a. To facilitate comparison, the total mass in all $\mathrm{K}$ contrast scenarios is identical. Figures $11 \mathrm{~b}$ and $11 \mathrm{c}$ show the concentration distributions for the $K_{\text {channel }} / K_{\text {matrix }}$ ratios of $10: 1$ and $30: 1$, respectively, at $t^{\prime}=0.25$. Compared with the corresponding concentration distributions resulting from the narrow source (Figures $2 \mathrm{c}$ and $2 \mathrm{~d}$ ), the plumes resulting from the partitioned source are similarly asymmetric and non-Gaussian with near-source peaks and extensive spreading (tailing) in the downgradient direction.

[49] Figure 12 shows the results of spatial moment analysis for the case in which the $K_{\text {channel }} K_{\text {matrix }}$ contrast was 10:1 for both channel network realizations. Compared to the narrow source case (Figure 3), the plume resulting from the partitioned source appears to have a stronger linear change with time in the spatial variance in the $-y$ direction. On the basis of Figure 12c, the dispersivities calculated from the temporal change in the second moments of concentration are $\alpha_{L}=0.25 \mathrm{~m}, \alpha_{T H}=0.0041 \mathrm{~m}$, and $\alpha_{T V}=0.0012 \mathrm{~m}$. These dispersivities, along with a molecular diffusion $8.64 \times 10^{-6} \mathrm{~m}^{2} / \mathrm{d}$, and a $v_{y}=0.0055 \mathrm{~m} / \mathrm{d}$, were used to produce the corresponding ADM.

[50] Figure 13 shows the plume skewness coefficients for different $K_{\text {channel }} / K_{\text {matrix }}$ contrasts at different relative times. After a sharp increase at early times, the calculated skewness coefficients decrease slowly. Compared with those resulting from the narrow source (Figure 4), the channel network plumes emanating from the partitioned source appear more symmetric for a given $K_{\text {channel }} / K_{\text {matrix }}$. Thus under the same $\mathrm{K}$ contrast the corresponding ADM plume resulting from a wide source is in better agreement with a channel network plume than the case in which the source is narrow. This is consistent with results from stochastic transport theory showing that a wide plume is more likely to be accurately simulated using an ADM.

[51] Figure 14 shows the vertically averaged concentrations from plumes in the channel network system overlain

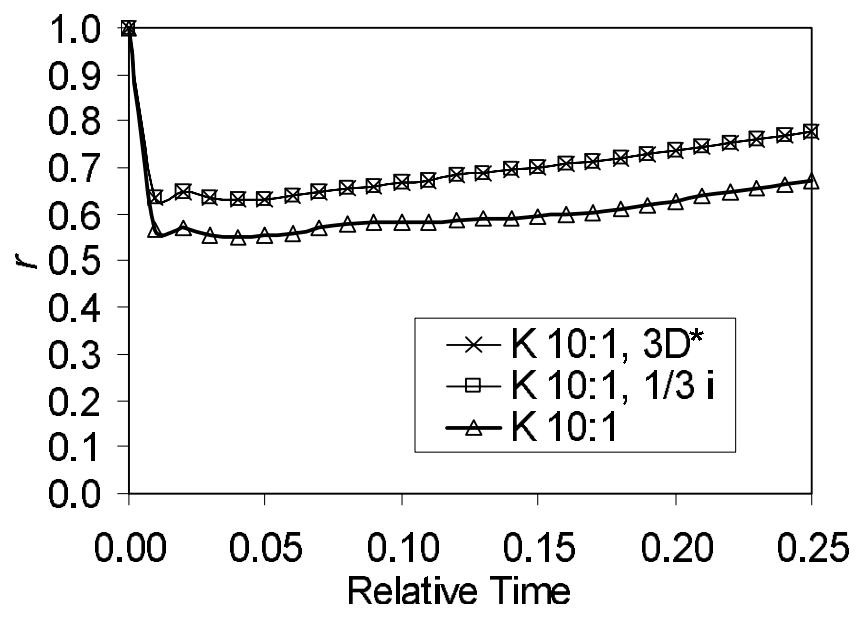

Figure 10. Correlation coefficient $r$ versus time in two numerical simulations for a $\mathrm{K}$ contrast of 10:1 in the narrow source case: one with diffusion coefficient $\left(D^{*}\right)$ increased 3 times and the other one with gradient (i) decreased to one third of the original value. Also shown are the $r$ values for the original base case. 
(a) Initial Partitioned Source

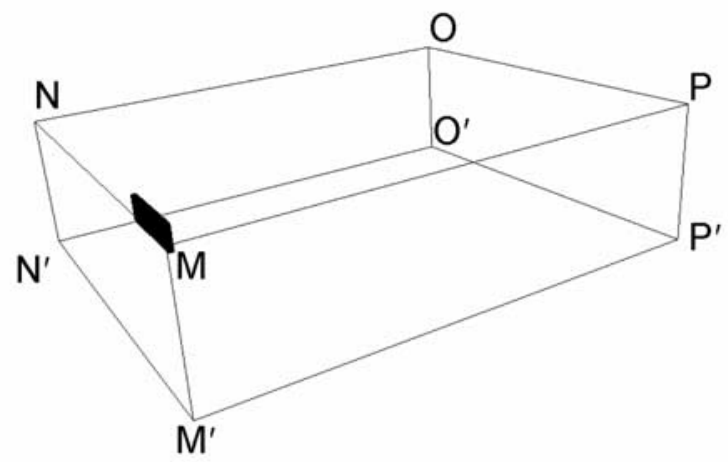

(b) K Ratio 10:1

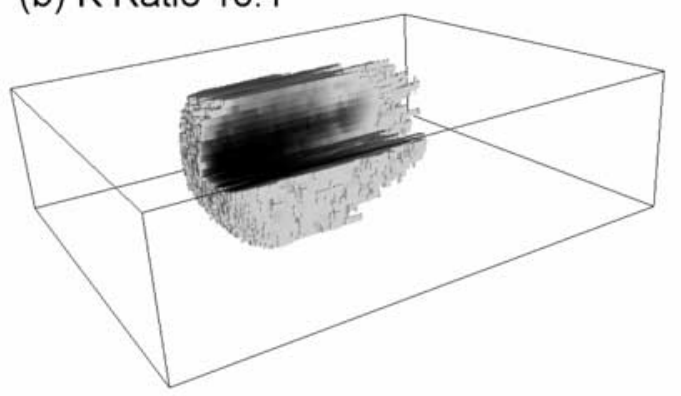

(c) K Ratio 30:1

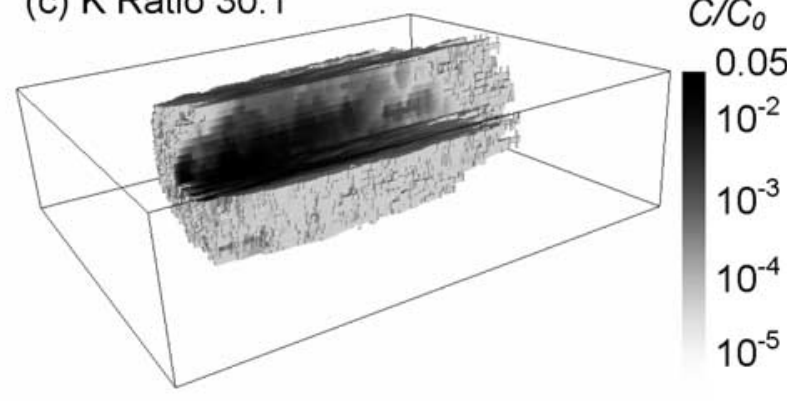

Figure 11. Calculated 3-D concentration distributions in the partitioned source case for different $\mathrm{K}$ contrast ratios $\left(K_{\text {channel }} / K_{\text {matrix }}\right)$ : (a) Source, (b) K ratio $10: 1$ at 0.25 pore volume, and (c) $\mathrm{K}$ ratio $30: 1$ at 0.25 pore volume. Concentrations are normalized by the initial source concentration. Vertical exaggeration is 5:1.

by contours from the corresponding ADM for different $K_{\text {channel }} / K_{\text {matrix }}$ contrasts. In each case, the corresponding ADM tracks the plume centers, but yields excessive upstream dispersion and underestimates downstream solute spreading. The larger the $K_{\text {channel }} / K_{\text {matrix }}$ contrast, the greater the discrepancy. Similar to the narrow source case, with increasing $K_{\text {channel }} / K_{\text {matrix }}$ contrasts the plumes in the channel network system exhibit successively greater departures from an ADM plume. The channel network versus ADM plume concentration residuals for the partitioned source case are plotted in Figure 15. The upstream residuals show a spatial structure similar to that of the ADM plume, and the downstream residuals are rather randomly distributed. The ADM provides a better match to the spatial structure of the channel network plumes in the downstream area.
[52] The channel network versus ADM plume correlation $r$ and mean fractional absolute residual (MFAR) are displayed in Figure 16 for different times and $K_{\text {channel }} / K_{\text {matrix }}$ contrasts. MFAR increases and $r$ decreases as the $K_{\text {channel }}$ $K_{\text {matrix }}$ contrast becomes large. The mismatch between the channel network and ADM plumes increases rapidly at early time and decreases gradually afterwards. When Figure 16 is compared to Figure 7, the magnitudes of MFAR and $r$ are quite different in the partitioned versus narrow source configuration cases. The fractional error, or the mismatch in spatial structure, associated with ADM is smaller in the partitioned source case. Results for realization 2 are shown in Figures 16c and 16d. The $r$ and MFAR values between the channel network and ADM plumes for the partitioned source at different $K_{\text {channel }} / K_{\text {matrix }}$ contrasts and relative times are similar to the results from realization 1. A summary of the major results from the second network realization can be found in Tables 1-3.

[53] It is clear from Figures 2, 5, 11, and 14 that trends in solute spreading and plume morphology depend on the nature of the source. The plume shape, and particularly the average longitudinal spreading, evolves based on two competing processes: advection along the channels and slow mass transfer to and from the matrix. For a narrow source, only after the initial mass is transferred into the high-K channels through slow mass transfer can it be quickly swept downstream by the enhanced advection in the channels. For the partitioned source, the large amount of initial channel source mass dominates plume evolution. Compared to the narrow source case, the slow mass transfer of initial mass out of low-K matrix does not control the plume shape.

[54] Suppose a correlation coefficient value of $r=0.7$ is the level above which the ADM produces a suitable representation of the plume migration through the channel network system. As seen in Figures 7 and 16, the acceptability of the ADM to portray transport through the channel network depends on three factors: transport time, the particular K contrast, and the source configuration. Accepting the $r=0.7$ cutoff, regardless of transport time the ADM can be used to represent transport through connected channel networks when the $\mathrm{K}$ contrast is less than $3: 1$ for both the narrow or partitioned source configurations. For dimensionless transport times exceeding 0.20 , the channelmatrix $\mathrm{K}$ contrast must be under $\sim 10: 1$ with the narrow source configuration, and under $\sim 20: 1$ with the partitioned source configuration for the ADM to provide an acceptable approximation. The ADM fails to reproduce transport behavior similar to that observed in the channel network system for all other conditions of transport times, $\mathrm{K}$ contrasts exceeding $\sim 20: 1$ or $\sim 30: 1$, and source configuration.

[55] As in the narrow source case, the two distinct transport behaviors upstream and downstream of plume centers are also evident in the partitioned source simulations (see Figures 14 and 15). The second spatial moments, dispersivities, and the mean fractional absolute residuals for the plume halves are summarized in Tables 2 and 3. Compared to the narrow source case, the upstream versus downstream contrasts in the indicators of asymmetric spreading are slightly smaller. This is due to the different initial channel mass in the two different source scenarios. The MFAR and $r$ values are also plotted in Figures $8 \mathrm{c}$ and 
(a) Zeroth Moment, Realization 1

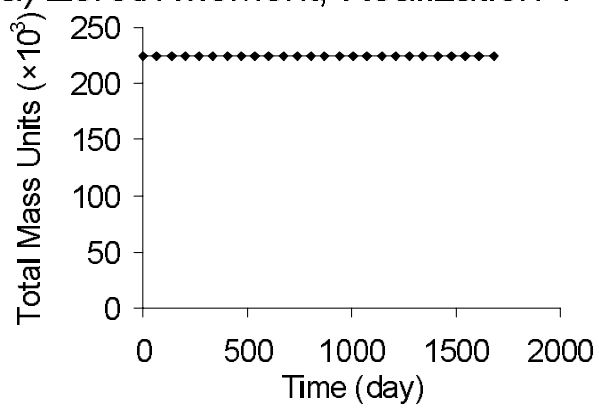

(c) Second Moments, Realization 1

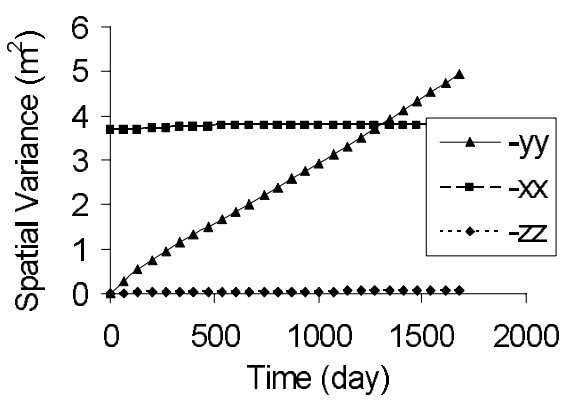

(e) Zeroth Moment, Realization 2

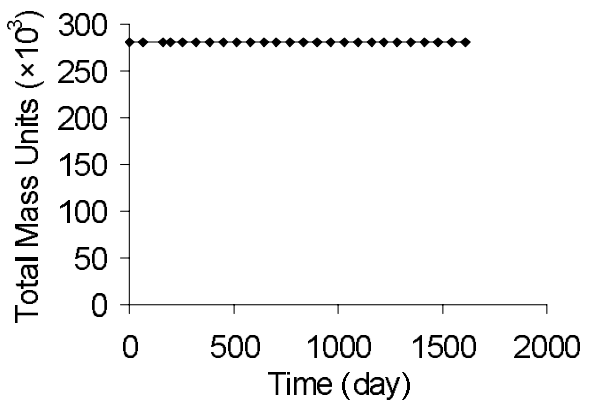

(g) Second Moments, Realization 2

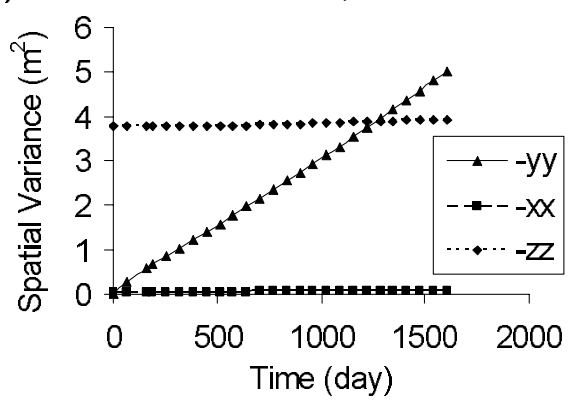

(b) First Moment, Realization 1

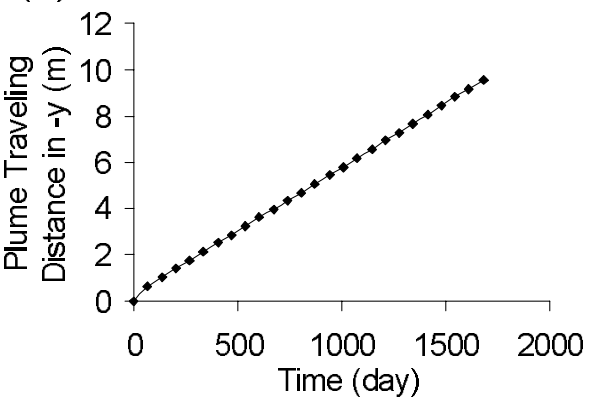

(d) Third Moments, Realization 1

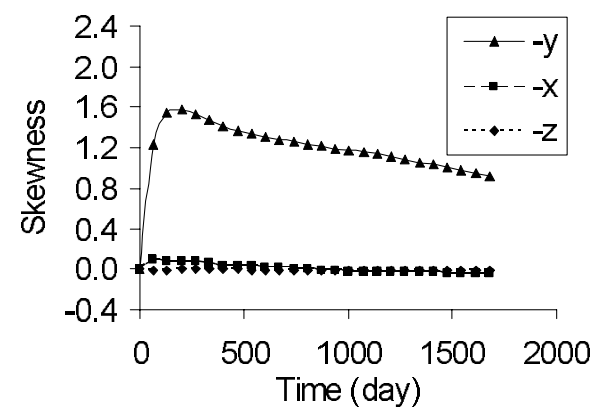

(f) First Moment, Realization 2

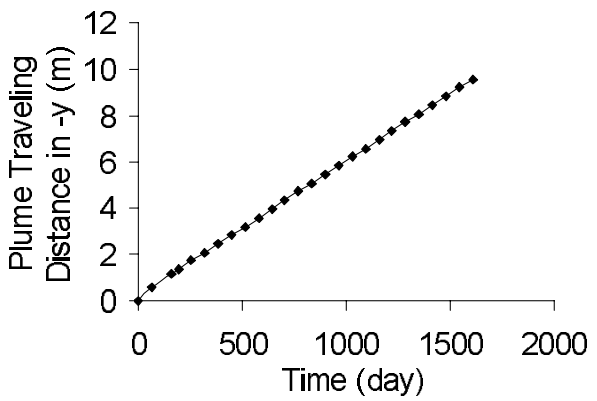

(h) Third Moments, Realization 2

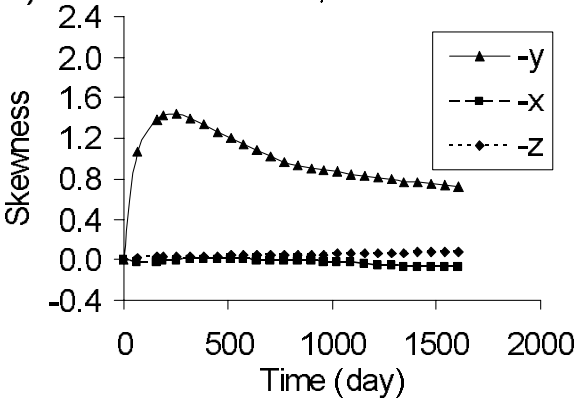

Figure 12. Spatial moment analysis results from the 3-D plumes in the channel network for a K contrast of 10:1 in the partitioned source case: (a and e) zeroth moment, ( $b$ and $\mathrm{f}$ ) first moment in ambient flow direction $y$, (c and $\mathrm{g}$ ) second moments in $-x x,-y y$, and $-z z$ directions, and ( $\mathrm{d}$ and $\mathrm{h}$ ) third moments in $-x,-y$, and $-z$ directions.

$8 \mathrm{~d}$ for different $\mathrm{K}$ contrasts and channel network realizations at the relative time 0.25 . Consistent trends were found across the narrow and partitioned source cases. It is noteworthy that plume correlations in narrow source cases, including full plume as well as plume halves, show some oscillations (see Figure 8). This is likely due to the difference in the widths of sources. The width of partitioned sources in both realizations is $7 \mathrm{~m}$, versus $0.4 \mathrm{~m}$ in realization 1 and $1.1 \mathrm{~m}$ in realization 2 for the narrow source cases. The wider the source, the larger number of throughgoing channels that dominate plume spreading. If the source is small relative to the representative channel spacing in the plume area (about $0.9 \mathrm{~m}$ ), plume behavior can be susceptible to the particular number of throughgoing channels.

[56] For the partitioned source configuration we confirmed the important role of diffusion and average flow velocity by conducting two additional tests: the diffusion 


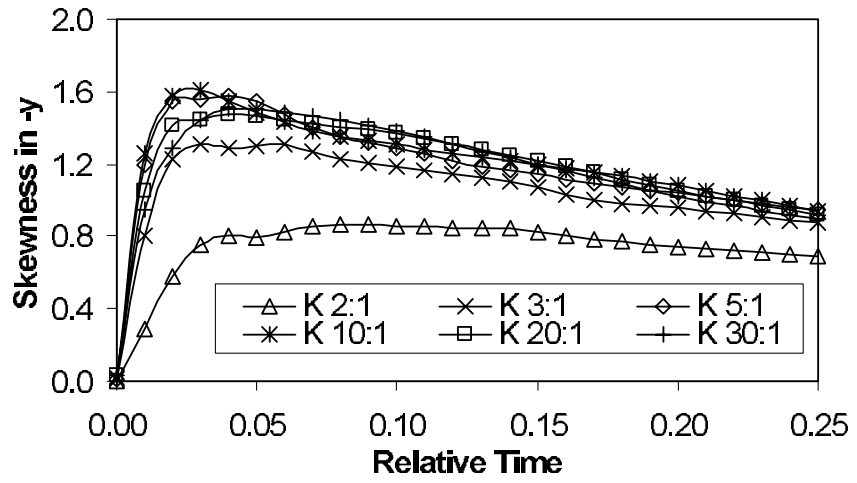

Figure 13. Plume skewness coefficients in the $-y$ direction for different $\mathrm{K}$ contrasts in the partitioned source case.

coefficient $\left(D^{*}\right)$ was increased 3 fold, and the hydraulic gradient, $i$, was decreased to one third of its original value. Similar to the narrow source, the rate of temporal change in the second moments of channel network plumes drops from $0.28 \times 10^{-2} \mathrm{~m}^{2} / \mathrm{d}$ in the base case to $0.24 \times 10^{-2} \mathrm{~m}^{2} / \mathrm{d}$ in the two additional tests. Figure 17 shows the correlation coefficient $r$ versus time for these two tests as well as the base case in which neither value was altered for a $\mathrm{K}$ contrast of 10:1. Here, as before, the two additional tests show no appreciable difference in the correlation coefficient values; however, when compared to the base case, the increase in diffusion noticeably enhances the correlation between the channel network and ADM plumes.

[57] To investigate the accuracy of using $10 \mathrm{~cm}$ wide cells to represent $10 \mathrm{~cm}$ wide channels in the numerical experiments, we conducted two additional tests each involving a mesh with smaller grid spacing. In one test we used a grid spacing of $5 \mathrm{~cm}$, and in the other test we used a grid spacing of $2.5 \mathrm{~cm}$. All other physical conditions remained identical to those in the original $10 \mathrm{~cm}$ grid case, and the channel width was maintained at $10 \mathrm{~cm}$. We compared the concentration distributions from these two additional finer-grid tests to those employing the original $10 \mathrm{~cm}$ grid. Despite some minor local smoothing observed using the $10 \mathrm{~cm}$ grid, the concentration distributions appeared to be quite similar when a finer grid was used. On the basis of these two additional finer-grid tests, the representation of $10 \mathrm{~cm}$ wide channels with $10 \mathrm{~cm}$ wide cells is considered sufficiently accurate for the purposes of this study.

\section{Summary and Conclusions}

[58] Stochastic transport theory has provided the concept of macrodispersion, which justifies field application of the advection-dispersion equation employing constant dispersivity values. The theory is founded on the randomizing effect of groundwater velocity variations induced by necessarily random, and perhaps spatially correlated, heterogeneity in hydraulic conductivity. Our results suggest the likely invalidity of the advective-dispersive model (ADM) employing the accepted macrodispersion concept when narrow connected pathways exist, even when heterogeneity is mild. We inspected how solute spreading is dictated by the presence of well-connected dendritic networks consisting of decimeter wide high-K channels. For a channel volume occupying only $9 \%$ of the total system, the effect of preferential flow was evident when the contrast in channel-matrix hydraulic conductivity was as low as $2: 1$. This contrast corresponds to a variance in $\log \mathrm{K}$ of merely 0.0075 , or variance in $\ln \mathrm{K}$ of 0.040 (see Table 1). Such heterogeneity in conductivity is mild by any realistic measure.

[59] Detailed 3-D simulations, involving complex channel network systems discretized into millions of $10 \mathrm{~cm}$ cells consistently showed that solute is transported forward of that predicted by the ADM applied to an equivalent system using an effective mean hydraulic conductivity. Plumes in the channel network systems were not Gaussian. The plumes exhibited significant asymmetry, with little solute dispersed upstream of the plume center, and extensive downstream spreading of low concentrations. For conditions of successively greater contrast in the channel-matrix $\mathrm{K}$ values, all of the latter features were enhanced.

[60] Use of the ADM is often justified based on temporal changes in the spatial statistics of solute concentrations. Suppose there is a field investigation in which concentration data exhibit no change in the zeroth moment indicating constant mass, the first moment is constant or otherwise physically reasonable given the velocity field, and the second moment shows a linear increase with time. With these observations in hand, one might presume that the $\mathrm{ADM}$ can be applied and dispersivities obtained from the second moment will be sufficient. Our results suggest that these spatial statistics of concentration are necessary but insufficient conditions to justify the application of the ADM.

[61] A key measure relied upon in macrodispersion theory is the second spatial moment of concentrations. We consistently observed a linear change in the second moment in all cases. In all cases, the $\mathrm{R}^{2}$ values of the line fit to the second moment of concentrations were greater than 0.97 . The corresponding ADM never represented the actual asymmetric spreading of the plume, particularly when the $\mathrm{K}$ contrast was "large". The reason that the second spatial moments of channel network plumes behave linearly with time is that the second moments of the two upstream and downstream halves each behave linearly. The ADM fails to describe the key behavior that upstream solute spreading is much less significant than downstream spreading. The dispersivities calculated for the downstream half plumes are consistently 2.1 to 2.8 times larger than the upstream ones. The ADM is not applicable when upstream and downstream spreading behaviors are so different.

[62] Unlike the second spatial moments, the third spatial moments, expressed in terms of skewness coefficients, can provide a more useful indicator on the potential applicability of macrodispersion theory. In all the cases examined in this study, the skewness coefficient ranged from $\sim 0.5$ for a $\mathrm{K}$ contrast of $2: 1$ to $\sim 1.0$ for a $\mathrm{K}$ contrast of $30: 1$. Given an instantaneous source, ADM-simulated plumes were symmetric or nearly so in the direction of flow, with the corresponding plume skewness close to zero. Thus, if the calculated skewness coefficient is large for an existing plume, the ADM is unlikely to provide an adequate representation. On the basis of the results of third moments, the $\mathrm{ADM}$ is inadequate to describe the asymmetry of plumes in 
(a) Source

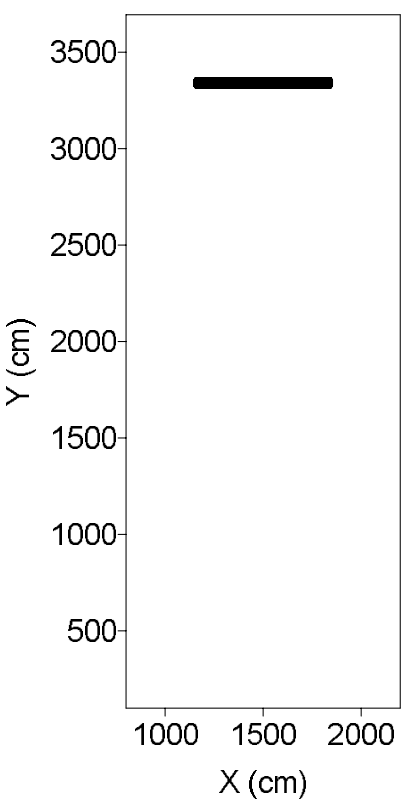

(b) $\mathrm{K} 2: 1$

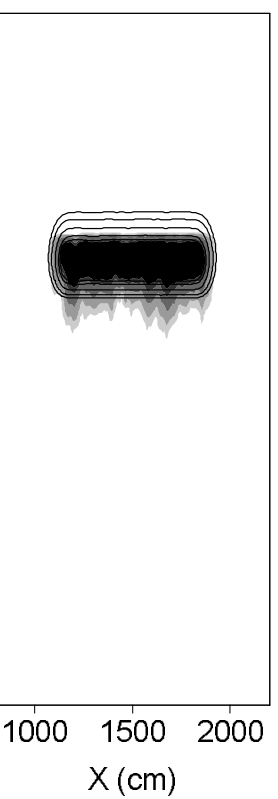

(c) K 3:1

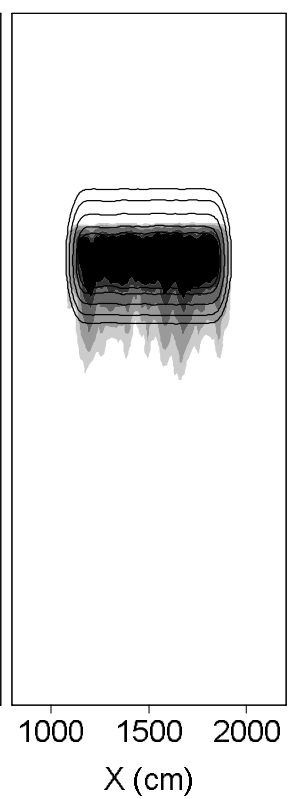

(d) $\mathrm{K} 5: 1$

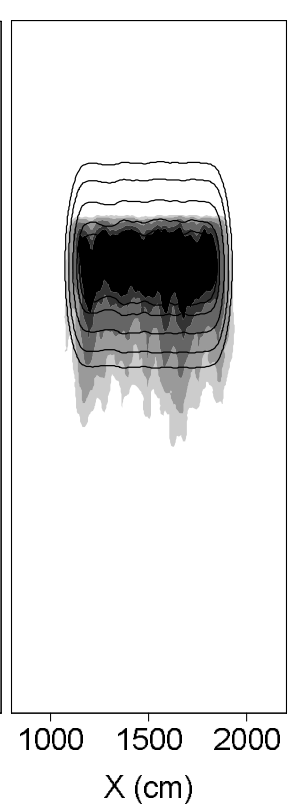

(e) K 10:1

(f) $\mathrm{K} 20: 1$

(g) $K 30: 1$
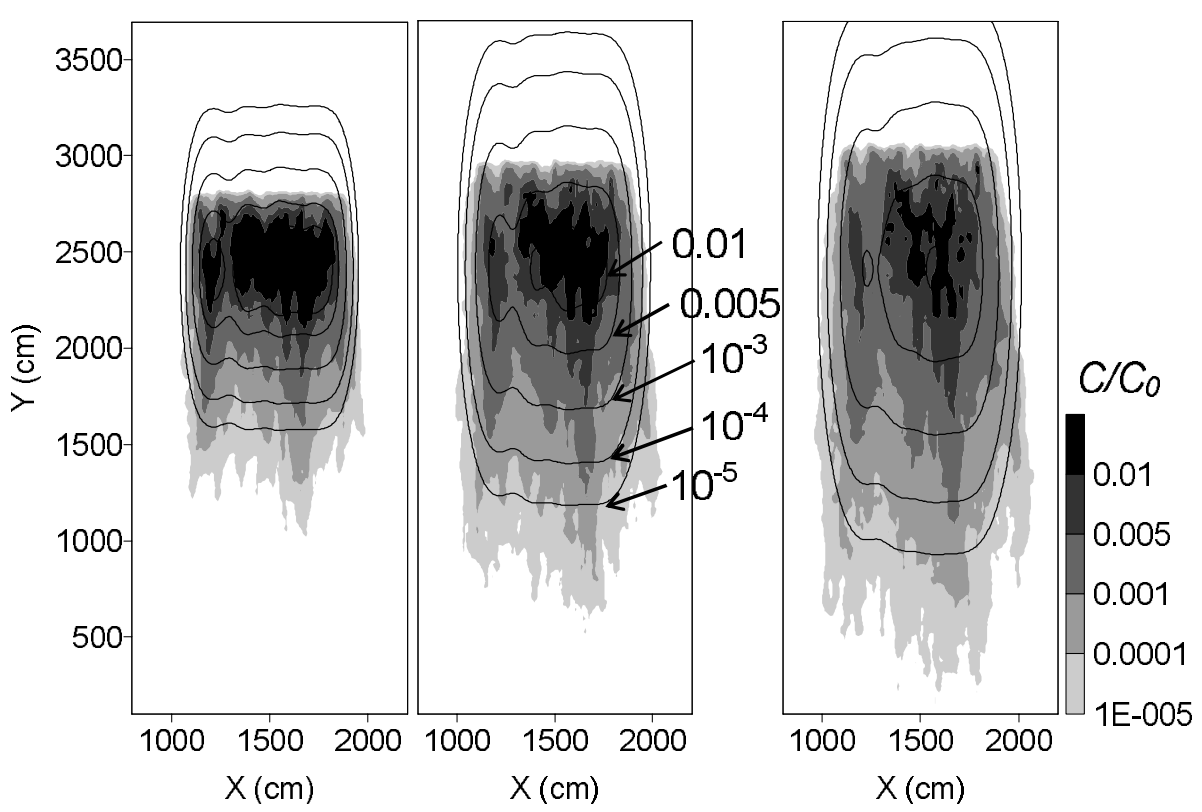

Figure 14. Comparison of the plumes in the channel network (shading) and from the corresponding ADM (contour lines) for different $\mathrm{K}$ contrasts in the partitioned source case: (a) Source, (b) K 2:1, (c) K 3:1, (d) K 5:1, (e) K 10:1, (f) K 20:1, and (g) K 30:1. Plumes are shown for a simulation time of 0.25 pore volume.

the channel network systems when the channel versus matrix $\mathrm{K}$ contrast was as low as $3: 1$.

[63] We quantitatively compared 3-D plumes in the channel network to 3-D plumes from the corresponding ADM. Two different measures, the mean fractional absolute residual, MFAR, and linear correlation coefficient $r$ were used. For all cases investigated, the correlation coefficient value was less than $\sim 0.7$ for systems with a binary $\mathrm{K}$ contrast of 20:1, which corresponds to a $\log K$ variance of 0.14 to 0.15 , or $\ln \mathrm{K}$ variance of 0.75 to 0.81 (see Table 1 ).
At early times the correlation of the plumes ranged from 0.45 to 0.50 , for the $20: 1 \mathrm{~K}$ contrast cases.

[64] If one is willing to accept the ADM as an approximation when it reproduces the plumes in the channel network system with a correlation of at least 0.7 , then certain guidance can be offered by our results. For the channel network systems when diffusion (and local subdecimeter-scale mechanical dispersion, if any) was dominated by advection, the primary determinants of applicability of the ADM were the channel-matrix $\mathrm{K}$ contrast and 
(a) $\mathrm{K} 2: 1$

(b) $\mathrm{K} 3: 1$

(c) K 5:1

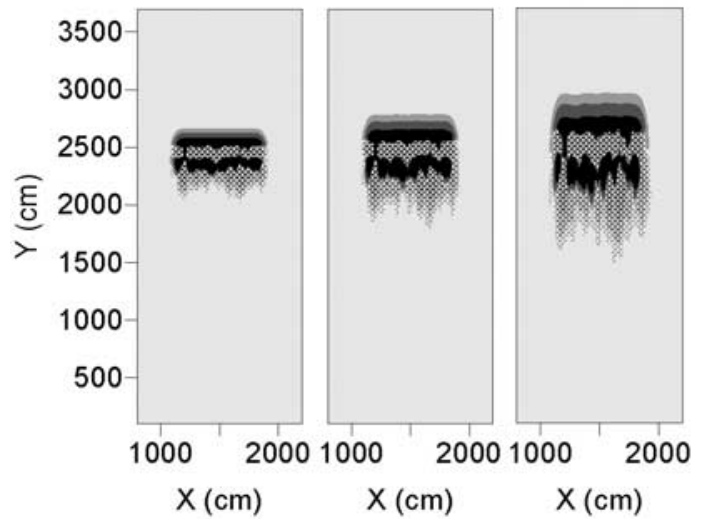

(d) $\mathrm{K}$ 10:1

(e) K 20:1

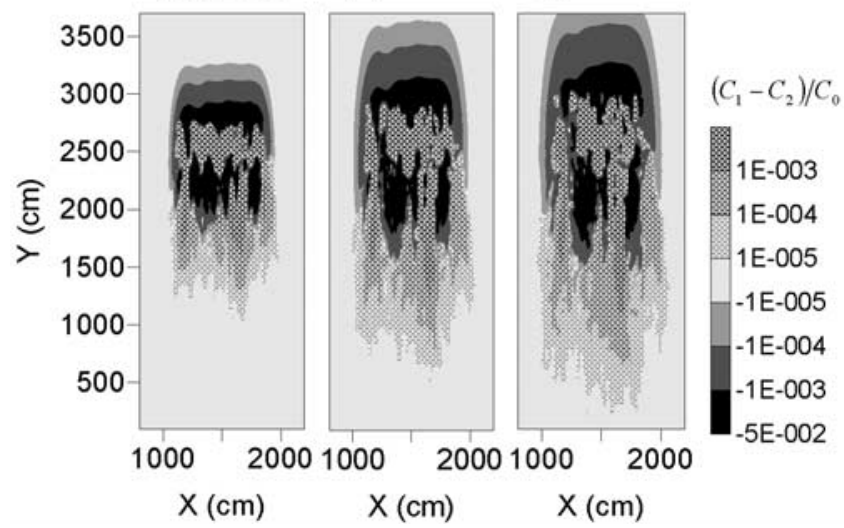

Figure 15. The network-ADM plume residuals for different $\mathrm{K}$ contrasts in the partitioned source case: (a) K 2:1, (b) K 3:1, (c) K 5:1, (d) K 10:1, (e) K 20:1, and (f) $\mathrm{K} 30: 1$ at 0.25 pore volume.

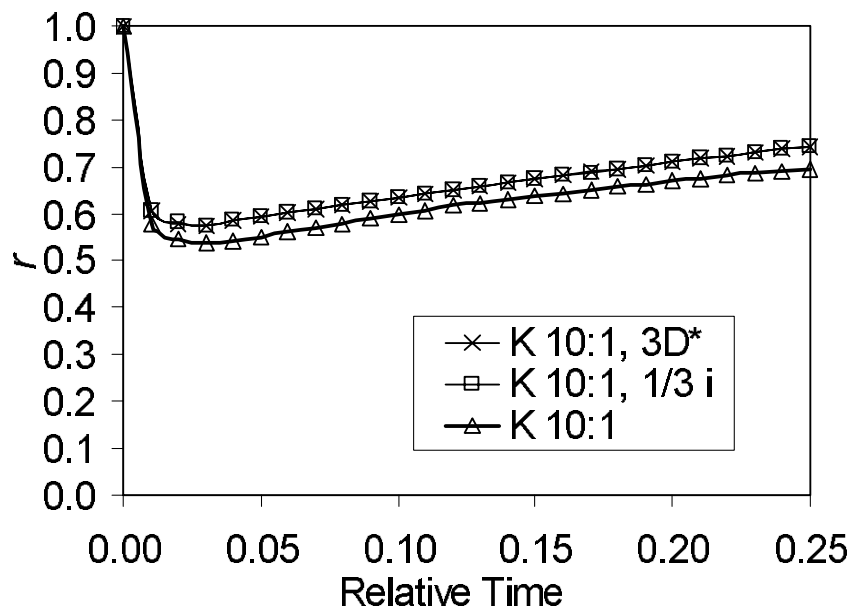

Figure 17. Correlation coefficient $r$ versus time in two numerical simulations for a $\mathrm{K}$ contrast of 10:1 in the partitioned source case: one with diffusion coefficient $\left(D^{*}\right)$ increased 3 times and the other one with gradient $(i)$ decreased to one third of the original value. Also shown are the $r$ values for the original base case.

whether or not the initial solute source was only in the matrix or in both the channels and matrix. When the source was contained in the matrix, the ADM was acceptable for $\mathrm{K}$ contrasts of up to $\sim 10: 1$, which is equivalent to a $\log \mathrm{K}$ variance of 0.083 to 0.091 , or $\ln \mathrm{K}$ variance of 0.44 to 0.48 . When the source was distributed in the matrix and channels in proportion to their conductivities, the ADM was acceptable for K contrasts up to 20:1, which is equivalent to a $\log \mathrm{K}$ variance of 0.14 to 0.15 or $\ln \mathrm{K}$ variance of 0.75 to 0.81 . These results are for transport (a) MFAR, Partitioned Source, Realization 1

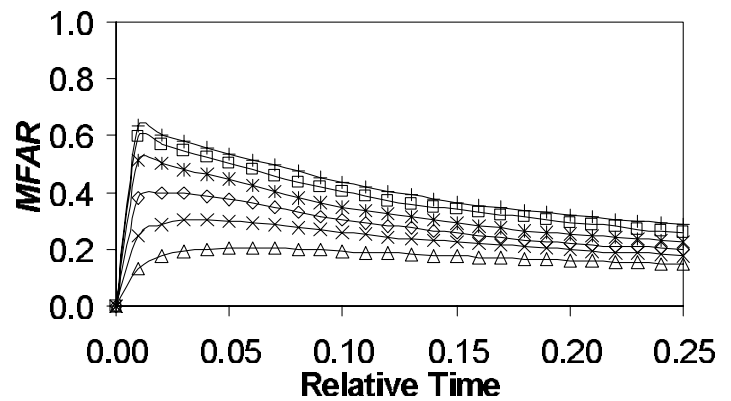

(c) MFAR, Partitioned Source, Realization 2

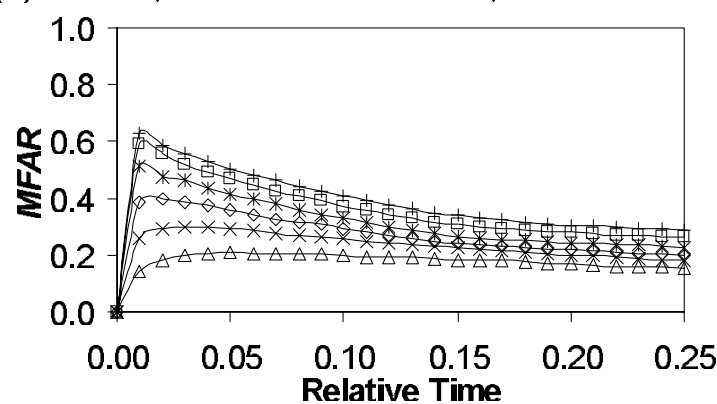

(b) $r$, Partitioned Source, Realization 1

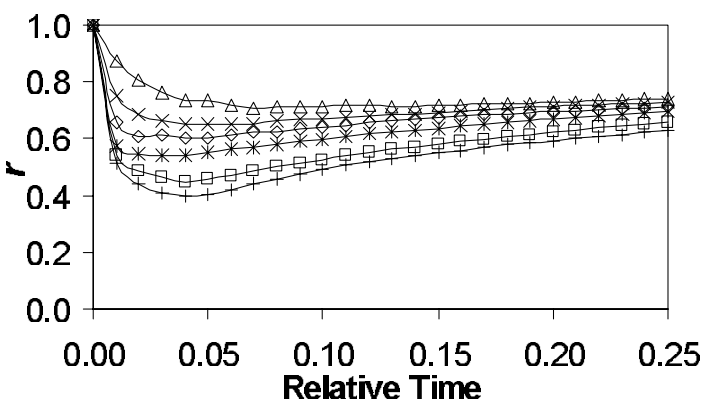

(d) $r$, Partitioned Source, Realization 2

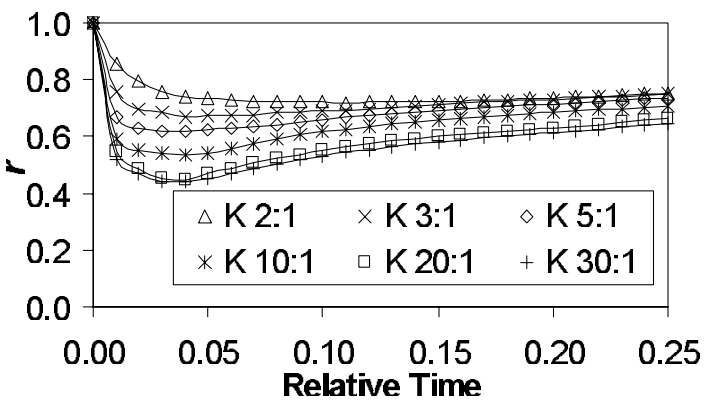

Figure 16. The MFAR and $r$ for different $\mathrm{K}$ contrasts in the partitioned source case: (a) MFAR, realization 1, (b) $r$, realization 1, (c) MFAR, realization 2, and (d) $r$, realization 2. The legend for different $\mathrm{K}$ contrasts is shown in Figure 16d. 
times corresponding to mean advective displacement at 25 percent of the system length. At early transport times the ADM was less applicable as indicated by a lower value of the correlation coefficient.

[65] Acknowledgments. This material is based upon work supported by the National Science Foundation under grants EAR-0003511 and EAR0003914. Any opinions, findings, and conclusions or recommendations expressed in this material are those of the author(s) and do not necessarily reflect the views of the National Science Foundation. Additional support was provided by the University of Alabama Graduate Council Fellowship and the Department of Geological Sciences Research Funds. The authors are grateful to the Associate Editor, an anonymous reviewer, and Brendan Zinn, whose constructive comments have led to an improvement of the manuscript. The authors also thank Paul Hsieh (USGS) for providing the 3-D visualization software used in this work.

\section{References}

Boggs, J. M., and E. E. Adams (1992), Field study of dispersion in a heterogeneous aquifer: 4. Investigation of adsorption and sampling bias, Water Resour. Res., 28(12), 3325-3336.

Boggs, J. M., L. M. Beard, S. E. Long, M. P. McGee, W. G. MacIntyre, C. P. Antworth, and T. B. Stauffer (1993), Database for the Second Macrodispersion Experiment (MADE-2), Tech. Rep. TR-102072, Electric Power Res. Inst., Palo Alto, Calif.

Burnett, R. D., and E. O. Frind (1987), An alternating direction Galerkin technique for simulation of groundwater contaminant transport in three dimensions: 2. Dimensionality effects, Water Resour. Res., 23(4), 695705 .

Carrera, J. (1993), An overview of uncertainties in modeling groundwater solute transport, J. Contam. Hydrol., 13, 23-48.

Dagan, G. (1984), Solute transport in heterogeneous porous formations, J. Fluid Mech., 145, 151-177.

Feehley, C. E., C. Zheng, and F. J. Molz (2000), A dual-domain mass transfer approach for modeling solute transport in heterogeneous porous media, application to the MADE site, Water Resour. Res., 36(9), 2051-2515.

Fogg, G. E., S. F. Carle, and C. Green (2000), Connected network paradigm for the alluvial aquifer system, in Theory, Modeling, and Field Investigation in Hydrogeology: A Special Volume in Honor of Shlomo P. Neuman's 60th Birthday, edited by D. Zhang and C. L. Winter, Spec. Pap. Geol. Soc. Am., 348, 25-42.

Freyberg, D. L. (1986), A natural gradient experiment on solute transport in a sand aquifer: 2. Spatial moments and the advection and dispersion of nonreactive tracers, Water Resour. Res., 22(13), 2031-2046.

Gelhar, L. W., A. L. Gutiahr, and R. L. Naff (1979), Stochastic analysis of macrodispersion in a stratified aquifer, Water Resour. Res., 15(6), 13871397.

Harbaugh, A. W., E. R. Banta, M. C. Hill, and M. G. McDonald (2000), MODFLOW-2000, the U.S. Geological Survey modular ground-water model-User guide to modularization concepts and the ground-water flow processes, U.S. Geol. Surv. Open File Rep., 00-92, 121 pp.
Harvey, C. F., and S. M. Gorelick (1995), Temporal moment-generating equations: Modeling transport and mass transfer in heterogeneous aquifers, Water Resour. Res., 31(8), 1895-1911.

Harvey, C. F., and S. M. Gorelick (2000), Rate-limited mass transfer or macrodispersion: Which dominates plume evolution at the Macrodispersion Experiment (MADE) site?, Water Resour. Res., 36(3), 637-650.

Julian, H. E., J. M. Boggs, C. Zheng, and C. E. Feehley (2001), Numerical simulation of a natural gradient tracer experiment for the natural attenuation study: Flow and physical transport, Ground Water, 39(4), 534-545.

Koltermann, C., and S. M. Gorelick (1996), Creating images of heterogeneity in sedimentary deposits: A review of descriptive, structureimitating, and process-imitating approaches, Water Resour. Res., 32(9), $2617-2658$

LaBolle, E. M., and G. E. Fogg (2001), Role of molecular diffusion in contaminant migration and recovery in an alluvial aquifer system, Transp. Porous Media, 42, 155-179.

Neuman, S. P., and Y. K. Zhang (1990), A quasi-linear theory of nonFickian and Fickian subsurface dispersion: 1. Theoretical analysis with application to isotropic media, Water Resour. Res., 26(5), 887-902.

Robin, M. J. L., A. L. Gutjahr, E. A. Sudicky, and J. L. Wilson (1993), Cross-correlated random field generation with the direct Fourier transform method, Water Resour. Res., 29(7), 2397-2835.

Rodriguez, I. I., and A. Rinaldo (1997), Fractal River Basins: Chance and Self-Organization, Cambridge Univ. Press, New York.

Stark, C. P. (1991), An invasion percolation model of drainage network evolution, Nature, 352, 423-425.

Tiedeman, C. R., and P. A. Hsieh (2004), Evaluation of longitudinal dispersivity estimates from simulated forced- and natural-gradient tracer tests in heterogeneous aquifers, Water Resour. Res., 40(1), W01512, doi:10.1029/2003WR002401.

Zheng, C., and S. M. Gorelick (2003), Analysis of solute transport in flow fields influenced by preferential flow paths at the decimeter scale, Ground Water, 4l(2), 142-155.

Zheng, C., and P. P. Wang (1999), MT3DMS: A modular three-dimensional multispecies model for simulation of advection, dispersion and chemical reactions of contaminants in groundwater systems; documentation and user's guide, Contract Rep. SERDP-99-1, U.S. Army Eng. Res. and Dev. Cent., Vicksburg, Miss.

Zinn, B., and C. F. Harvey (2003), When good statistical models of aquifer heterogeneity go bad: A comparison of flow, dispersion, and mass transfer in connected, and multivariate Gaussian hydraulic conductivity fields, Water Resour. Res., 39(3), 1051, doi:10.1029/2001WR001146.

S. M. Gorelick, Department of Geological and Environmental Sciences, Stanford University, Stanford, CA 94305, USA.

G. Liu and C. Zheng, Department of Geological Sciences, University of Alabama, 202 Bevill Building, Box 870338, Tuscaloosa, AL 35487, USA (czheng@ua.edu) 